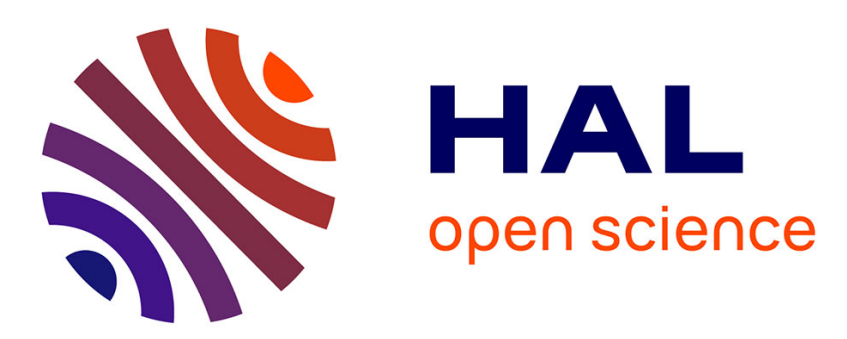

\title{
Etude comparative de quelques réfrigérateurs solaires utilisant une réaction chimique entre un solide et un gaz M. Balat
}

\section{To cite this version:}

M. Balat. Etude comparative de quelques réfrigérateurs solaires utilisant une réaction chimique entre un solide et un gaz. Revue de Physique Appliquée, 1989, 24 (6), pp.671-689. 10.1051/rphysap:01989002406067100 . jpa-00246092

\section{HAL Id: jpa-00246092 https://hal.science/jpa-00246092}

Submitted on 1 Jan 1989

HAL is a multi-disciplinary open access archive for the deposit and dissemination of scientific research documents, whether they are published or not. The documents may come from teaching and research institutions in France or abroad, or from public or private research centers.
L'archive ouverte pluridisciplinaire HAL, est destinée au dépôt et à la diffusion de documents scientifiques de niveau recherche, publiés ou non, émanant des établissements d'enseignement et de recherche français ou étrangers, des laboratoires publics ou privés. 


\title{
Etude comparative de quelques réfrigérateurs solaires utilisant une réaction chimique entre un solide et un gaz
}

\author{
M. Balat \\ Institut de science et de génie des Matériaux et Procédés, IMP-CNRS, UP 32, BP 5 Odeillo, 66120 Font- \\ Romeu, France
}

(Reçu le 25 novembre 1988, révisé et accepté le 17 février 1989)

Article de mise au point

Résumé. - C'est dans l'objectif de la production de glace par voie solaire, et avec des systèmes utilisant une réaction entre un solide et un gaz que nous avons fait cette étude bibliographique. Nous comparerons, à partir de différents critères, les systèmes développés par les auteurs et nous y ajouterons les performances obtenues sur notre prototype de réfrigérateur solaire.

Abstract. - In the aim of ice production by solar mean and with systems using a chemical reaction between a solid and a gas, we have done this bibliographical study. With several criterions, we will compare the systems developped by the authors and we will add our results obtained on our prototype of solar refrigerator.

\section{Introduction.}

Différents systèmes ont été utilisés pour la réfrigération solaire dont les plus connus sont :

- l'absorption liquide-gaz (système divariant à l'équilibre) en cycle intermittent : eau $/ \mathrm{NH}_{3}$;

- l'adsorption solide-gaz (système divariant à l'équilibre); zéolithe/eau ou charbon actif/méthanol ;

- la réaction chimique entre un solide et un gaz (système monovariant à l'équilibre): $\mathrm{CaCl}_{2} / \mathrm{NH}_{3}$ par exemple.

Une étude bibliographique comparative de ces différents principes montre l'avantage des réactions solide-gaz du point de vue de la densité énergétique présentée sous la forme d'un rapport masse de gaz cyclée sur masse de solide (ou de solution pour l'adsorption liquide-gaz), de la puissance extraite à l'évaporateur en $\mathrm{W} / \mathrm{kg}$ de solide (ou de solution) ainsi que du rapport masse de glace sur masse de solide (ou de solution) (cf. Tab. I).

Tableau I. - Comparaison des trois principaux systèmes de réfrigération.

[Comparison of the three principal refrigeration systems.]

\begin{tabular}{|l|c|c|c|}
\hline & $\frac{\text { masse gaz cyclée }}{\text { masse de sol. ou liq. }}$ & $\frac{\text { masse de glace }}{\text { masse de sol. ou liq. }}$ & $\begin{array}{c}P \text { extraite à l'évap. } \\
\text { W/kg de sol. ou liq. }\end{array}$ \\
\hline $\begin{array}{c}\text { Absorption } \\
\text { liquide-gaz [1-6] }\end{array}$ & 0,09 & 0,08 & 0,5 \\
\hline $\begin{array}{c}\text { Adsorption } \\
\text { solide-gaz [7-10] }\end{array}$ & 0,13 & 0,26 & 2 à 3 \\
\hline $\begin{array}{l}\text { Réaction } \\
\text { solide-gaz [11-34, 38,39] }\end{array}$ & 0,70 & 1 & 10 à 20 \\
\hline
\end{tabular}




\section{Systèmes divariants à l'équilibre : absorption et} adsorption.

Nous allons présenter brièvement l'absorption liquide-gaz et l'adsorption solide-gaz.

1.1 ABSORPTION LIQUIDE-GAZ EN CYCLE INTERMITTENT. - Toute machine à absorption nécessite trois sources thermiques :

- une source froide (milieu à réfrigérer) à laquelle on doit prélever de la chaleur;

- une source intermédiaire (milieu ambiant) qui reçoit de la chaleur;

- une source chaude (système de captation de l'énergie solaire) qui fournit l'énergie consommée par la machine et quatre appareils sont nécessaires pour permettre d'échanger l'énergie thermique entre le couple solvant-réfrigérant et les trois sources thermiques : le bouilleur, le condenseur, l'évaporateur et l'absorbeur. Bouilleur et absorbeur peuvent être un seul et même appareil et le schéma de base de la machine à absorption discontinue se réduit alors (Fig. 1).

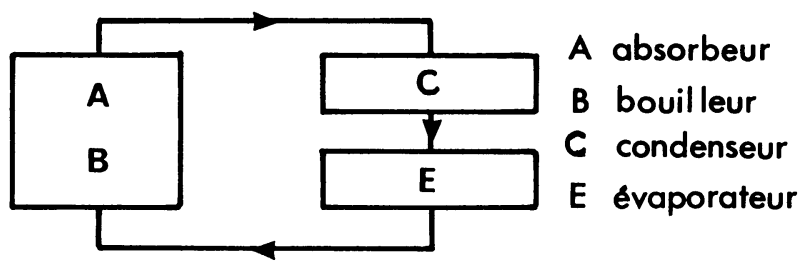

Fig. 1. - Schéma de la machine à absorption liquide-gaz discontinue.

[Sketch of the liquid-gas absorption machine (batch functioning).]

Dans un tel type de machine à absorption, le choix du couple solvant-réfrigérant est déterminé par de nombreux critères de sélection dont :

- une zone de solubilité et d'équilibre couvrant le plus grand domaine de titres et de températures possible ;

- une différence de volatilité entre les deux constituants la plus grande possible pour éviter l'utilisation d'une colonne de rectification;

- des zones de congélation ou de cristallisation éloignées des conditions normales et transitoires de fonctionnement ;

- des niveaux de pression au condenseur et à l'évaporateur techniquement acceptables (il faut éviter l'emploi de techniques du vide ou des hautes pressions) ;

- une faible viscosité des solutions ;

- une forte chaleur latente de vaporisation du réfrigérant ;

— des solutions à faibles corrosivité et toxicité, ainsi qu'une bonne stabilité chimique des constituants dans la gamme de températures utilisée ;

- des constituants peu coûteux.

Parmi les réalisations de réfrigérateurs basés sur le principe de l'absorption, on rencontre essentiellement trois types de couples: $\mathrm{NH}_{3} / \mathrm{H}_{2} \mathrm{O}$, $\mathrm{CH}_{3} \mathrm{NH}_{2} / \mathrm{LiSCN}$ ou $\mathrm{NaSCN}$ et $\mathrm{NH}_{3} / \mathrm{NaSCN}$.

Dans le tableau II, nous présentons les résultats obtenus par différents auteurs, avec le couple $\mathrm{NH}_{3} / \mathrm{H}_{2} \mathrm{O}$, celui-ci étant le plus utilisé.

Les performances des machines sont calculées à partir des quantités de chaleur suivantes :

$$
\begin{aligned}
Q_{\mathrm{N}}= & \text { chaleur latente d'évaporation du gaz } Q_{\mathrm{E}} \\
& \text { - pertes } Q_{\mathrm{P}} \\
= & \text { froid net produit }
\end{aligned}
$$

$Q_{D^{\prime}}=$ quantité de chaleur nécessaire pour effectuer la désorption $Q_{\mathrm{D}}+$ chaleur sensible $Q_{\mathrm{CS}}$ (réacteur, solution...)

$Q_{\mathrm{I}}=$ énergie incidente totale

$Q_{\mathrm{G}}=$ quantité de chaleur correspondant à la masse de glace formée $\times$ chaleur latente de fusion de la glace à $0^{\circ} \mathrm{C}$.

Les C.O.P. exprimés dans le tableau II sont alors :

- pour les C.O.P. thermiques :

$$
\text { C.O.P.th }{ }_{\text {th }}=Q_{\mathrm{N}} / Q_{\mathrm{D}^{\prime}} \quad \text { C.O.P.th } \text { th }_{\mathrm{G}}=Q_{\mathrm{D}^{\prime}}
$$

- pour les C.O.P. solaires :

$$
\text { C.O.P. } \text { sol N }=Q_{\mathrm{N}} / Q_{\mathrm{I}} \quad \text { C.O.P. } \text { sol G }=Q_{\mathrm{G}} / Q_{\mathrm{I}} \text {. }
$$

L'efficacité $\boldsymbol{\eta}$ du capteur est alors définie par :

$$
\eta=Q_{\mathrm{D}^{\prime}} / Q_{\mathrm{I}}=\text { C.O.P. } \cdot_{\text {sol N }} / \text { C.O.P.th N }
$$

Dans tous les cas, la production de glace (exprimée en $\mathrm{kg} /$ jour et par $\mathrm{m}^{2}$ de capteur) est très faible, sauf dans le cas de l'expérience de Trombe [2] pour laquelle les capteurs utilisés sont à concentration, l'eau mise au départ dans les bacs en contact avec l'évaporateur est à une température de $4{ }^{\circ} \mathrm{C}$ et les températures de condensation sont basses, ce qui tend à augmenter notablement les performances de la machine.

La seule machine produisant une quantité de glace mesurable est celle réalisée en 1962 par Chinnappa [3]. Une autre réalisation, celle du « Laboratory for Refrigeration Engineering " [6] a conduit à une production de 2 à $2,5 \mathrm{~kg}$ de glace par jour et par $\mathrm{m}^{2}$, avec un seul cycle journalier mais les C.O.P. n'ont pu être calculés par manque de données.

En conclusion, le système $\mathrm{NH}_{3} / \mathrm{H}_{2} \mathrm{O}$ en cycle intermittent permet de produire de la glace dans des conditions de températures précises imposées par un climat tropical $\left(T_{\text {jour }}=35-40^{\circ} \mathrm{C}, T_{\text {nuit }}=30^{\circ} \mathrm{C}\right)$, mais toutes les expériences réalisées donnent des quanti- 
Tableau II. - Comparaison des performances obtenues par différents auteurs sur le couple $\mathrm{H}_{2} \mathrm{O} / \mathrm{NH}_{3}$ (absorption liquide-gaz).

[Comparison of the performances obtained by different authors on the pair $\mathrm{H}_{2} \mathrm{O} / \mathrm{NH}_{3}$ (liquid-gas absorption).]

\begin{tabular}{|c|c|c|c|c|c|c|c|c|}
\hline Auteurs & $\begin{array}{l}\text { Maillet } \\
\text { (th.) } \\
\end{array}$ & $\begin{array}{l}\text { Maillet } \\
\text { (exp.) }\end{array}$ & $\begin{array}{r}\text { Trombe } \\
\left(1,5 \mathrm{~m}^{2}\right) \\
(1\end{array}$ & $\begin{array}{l}\text { Trombe } \\
\left(18 \mathrm{~m}^{2}\right) \\
\text { 8) }\end{array}$ & $\begin{array}{c}\text { Chinnappa } \\
\text { (1962) }\end{array}$ & $\begin{array}{l}\text { Duffie } \\
\text { (1958) }\end{array}$ & $\begin{array}{c}\text { Venkatesh } \\
\text { (1979) }\end{array}$ & $\begin{array}{c}\text { Van Paassen } \\
\text { (1985) }\end{array}$ \\
\hline $\begin{array}{c}T \text { des }\left({ }^{\circ} \mathrm{C}\right) \\
\text { solưtion }\end{array}$ & 90 & 72 & 80 & 90 & 100 & $125 / 130$ & 103 & 90 \\
\hline$T$ cond. $\left({ }^{\circ} \mathrm{C}\right)$ & 40 & 23 & 15 & $<10$ & 32 & $25 / 30$ & 32 & 25 \\
\hline$T$ abs. $\left({ }^{\circ} \mathrm{C}\right)$ & 40 & - & - & - & $35 / 37$ & - & 44 & 30 \\
\hline$T$ évap. $\left({ }^{\circ} \mathrm{C}\right)$ & 0 & 1,5 & - & - & $-10 /-7$ & 0 & 6 & $-10 /-5$ \\
\hline$P_{\mathrm{h}}($ bar $)$ & 16 & 10 & 7,5 & $<6,5$ & 13 & $10 / 12$ & 13 & 10 \\
\hline$P_{\mathrm{b}}(\mathrm{bar})$ & 4,5 & 5 & - & - & $3 / 4$ & 4,5 & 6 & $3 / 3,5$ \\
\hline$t_{\text {dés. }}(\mathrm{h})$ & - & 7 & 6 & 7,5 & 5,5 & 2,5 & - & - \\
\hline$t_{\text {abs. }}(\mathrm{h})$ & - & 11 & - & - & 5 & - & 8 & 8 \\
\hline $\begin{array}{c}m \mathrm{NH}_{3}(k g) \\
\text { cyclée }\end{array}$ & 19,1 & 2,4 & 2,5 & 44 & 1,2 & - & 1,1 & 3 \\
\hline$Q_{\mathrm{I}}(\mathrm{MJ})$ & - & 40 & 21,6 & 356,4 & 21,1 & - & 15,9 & - \\
\hline$Q_{\mathrm{N}}(\mathrm{MJ})$ & 20,5 & 2,37 & $\begin{aligned}< & 3,4 \\
& \text { (sans }\end{aligned}$ & $\begin{array}{l}<60,2 \\
\text { ertes) }\end{array}$ & 1,28 & 0,94 & 1,36 & - \\
\hline$m_{\mathrm{g}}\left(\mathrm{kg} \cdot \mathrm{m}^{-2} \cdot \mathrm{jr}^{-1}\right)$ & - & 0 & $4(*)$ & $5,5(*)$ & 1,4 & - & 0 & $2 / 2,5$ \\
\hline$Q_{\mathrm{D}^{\prime}}(\mathrm{MJ})$ & 147,7 & 16,8 & - & - & 7,16 & - & - & - \\
\hline C.O.P. th N & 0,139 & 0,141 & - & - & 0,179 & - & - & - \\
\hline C.O.P.th G & - & 0 & - & - & 0,106 & - & 0 & - \\
\hline C.O.P. $\cdot_{\text {sol N }}$ & - & 0,059 & $\begin{array}{r}<0,157 \\
\quad \text { (cor }\end{array}$ & $\begin{array}{l}<0,169 \\
\text { nt.) }\end{array}$ & 0,061 & - & 0,086 & - \\
\hline C.O.P. sol G & - & 0 & $\begin{array}{r}0,093 \\
\quad \text { (cor }\end{array}$ & nt.) & 0,035 & - & 0 & - \\
\hline$\eta$ & - & 0,42 & - & - & - & 0,35 & - & - \\
\hline
\end{tabular}

(*) $T_{\text {cau }}$ initiale $=4^{\circ} \mathrm{C}$.

tés de glace allant de 1 à $2,5 \mathrm{~kg}$ par jour et par $\mathrm{m}^{2}$ de capteur inférieures à celles qui peuvent être obtenues, dans les mêmes conditions de températures, par l'utilisation de systèmes à absorbant solide (absorption avec charbon actif/méthanol, ou réaction chimique).

En outre, ces systèmes nécessitent d'importants volumes de solution au bouilleur-absorbeur pour de faibles quantités de gaz cyclées, leur densité énergétique est donc faible. C'est un inconvénient que l'on ne rencontre pas dans les systèmes basés sur une réaction solide-gaz.

1.2 LE CYCLE INTERMITTENT À ADSORPTION SOLIDE-GAZ. - La réfrigération basée sur le principe de l'adsorption met en jeu un fluide qui se fixe à la surface d'un solide par des liaisons de faible énergie ; cette fixation n'entraînant aucune modification macroscopique du solide (gonflement, déformation...) sauf une variation de sa masse. Ce principe est analogue à l'absorption dite liquide mis à part le fait que l'un des deux constituants est solide, ceci entraînant la suppression de la colonne de rectification.

Dans le cadre de la réfrigération solaire, les couples les plus étudiés sont les couples zéolithe $13 \mathrm{X} / \mathrm{H}_{2} \mathrm{O}$ (pour une température d'évaporation $\geqslant 0{ }^{\circ} \mathrm{C}$ ) et charbon actif $35 /$ méthanol (pour une température d'évaporation $\leqslant 0{ }^{\circ} \mathrm{C}$ ).

Trois réalisations ont été faites par l'équipe de Meunier [7-10] à Orsay. Il s'agit de :

- la glacière solaire [7] dont la production moyenne de glace dans l'évaporateur (glace non récupérable) est de $6,3 \mathrm{~kg}$ par $\mathrm{m}^{2}$ de capteur et par jour, pour une masse de zéolithe de $23 \mathrm{~kg}$. Cette glace sert à maintenir une température de 3 à $7{ }^{\circ} \mathrm{C}$ dans la glacière de 150 l. Le C.O.P. solaire (rapport $\mathrm{du}$ froid net produit sur l'énergie incidente totale) est de 0,095 ;

- l'entrepôt frigorifique [8] de $12 \mathrm{~m}^{3}$ produisant de la glace pour le stockage de froid, avec un C.O.P. solaire de 0,096 ; la masse de zéolithe de $305 \mathrm{~kg}$ est répartie dans 24 capteurs de surface totale $20,3 \mathrm{~m}^{3}$; - la machine à glace $[9,10]$, basée sur le couple 
charbon actif $35 /$ méthanol, permet elle de produire de la glace récupérable dont la production varie de 4 à $6 \mathrm{~kg}$ par jour et par $\mathrm{m}^{2}$, avec un C.O.P. solaire variant de 0,070 à 0,120 .

Dans tous ces systèmes, l'adsorbant est situé directement dans des capteurs plans portant un revêtement sélectif au chrome noir. Condenseur et évaporateur sont deux appareils distincts ; le condenseur est de type à eau dans le cas de l'entrepôt, et du type à air dans le cas de la glacière et de la machine à glace.

Dans le tableau III, sont comparées les performances et les caractéristiques de ces 3 prototypes. La lecture de ce tableau appelle quelques remarques :

- la densité énergétique de ces systèmes est très faible. En effet, la quantité de gaz cyclée (en $\mathrm{kg} / \mathrm{kg}$ de solide) est de : 0,062 pour le couple zéolithe $13 \mathrm{X} / \mathrm{H}_{2} \mathrm{O}, 0,133$ pour le couple charbon actif/méthanol ;
- les pressions de fonctionnement sont très faibles, ce qui ne permet pas l'utilisation de clapets anti-retour à la place des vannes. D'autre part, l'étanchéité du montage doit être parfaite car il règne dans les canalisations une pression toujours très inférieure à la pression atmosphérique.

La puissance exprimée dans le tableau III est celle qui correspond à la production de froid net (chaleur latente d'évaporation - pertes) pendant la durée de l'adsorption (environ $12 \mathrm{~h}$ ) et elle est exprimée en $\mathrm{W} / \mathrm{kg}$ d'adsorbant. Cette puissance est faible dans le cas du couple zéolithe $13 \mathrm{X} / \mathrm{H}_{2} \mathrm{O}$ puisque la quantité de gaz cyclée par unité de masse d'adsorbant est plus faible dans ce cas-là.

Les C.O.P. définis dans ce tableau sont les mêmes que ceux définis dans le tableau II (où $\left.Q_{\mathrm{D}^{\prime}}=D_{\mathrm{D}}+Q_{\mathrm{CS}}\right)$.

Les systèmes à adsorption présentent donc des avantages (C.O.P. élevés, températures d'utilisation

Tableau III. - Comparaison des performances des trois prototypes basés sur l'adsorption solide-gaz.

[Comparison of the performances of the three prototypes based on solid-gas adsorption.]

\begin{tabular}{|c|c|c|c|}
\hline $\begin{array}{l}\text { Couple } \\
\text { Prototype } \\
\text { [Réf.] }\end{array}$ & $\begin{array}{c}\text { Zéolith } \\
\text { glacière solaire } \\
\text { [7] }\end{array}$ & $\begin{array}{l}13 \mathrm{X} / \mathrm{H}_{2} \mathrm{O} \\
\text { entrepôt frigorifique } \\
{[8]}\end{array}$ & $\begin{array}{c}\text { CA35/méthanol } \\
\text { machine à glace } \\
{[9]}\end{array}$ \\
\hline $\begin{array}{l}T \text { dés. } / T \text { cond. }\left({ }^{\circ} \mathrm{C}\right) \\
T_{\mathrm{f}} \text { ads. } / T \text { évap. }\left({ }^{\circ} \mathrm{C}\right) \\
P \text { cond. } / P \text { évap. }(\mathrm{mbar})\end{array}$ & $\begin{array}{c}111 / 31 \\
23 / 0 \\
60 / 6\end{array}$ & $\begin{array}{c}117-137 / 32 \\
14 / 0 \\
60 / 6\end{array}$ & $\begin{array}{l}85-95 /- \\
-/-3 \\
350 / 30\end{array}$ \\
\hline $\begin{array}{l}m \text { adsorbant } \\
\text { solide }(\mathrm{kg}) \\
m \text { gaz cyclée }(\mathrm{kg})\end{array}$ & $\begin{array}{l}23,3 \\
0,854\end{array}$ & $\begin{array}{l}305 \\
18,9\end{array}$ & $\begin{array}{r}135 \\
18\end{array}$ \\
\hline $\begin{array}{l}Q_{\mathrm{E}}(\mathrm{MJ}) \\
Q_{\mathrm{I}}(\mathrm{MJ}) \\
Q_{\mathrm{Cs}}(\mathrm{MJ}) \\
Q_{\mathrm{D}}(\mathrm{MJ}) \\
Q_{\mathrm{N}}(\mathrm{MJ}) \\
P(\mathrm{~W} / \mathrm{kg} \text { ads. })\end{array}$ & $\begin{array}{r}2 \\
19,9 \\
3,8 \\
2,7 \\
1,9 \\
1,9\end{array}$ & $\begin{array}{c}45,3 \\
469 \\
137,3 \\
45,3 \text { (sans pertes) } \\
3,44\end{array}$ & $\begin{array}{c}19,8 \\
120 \\
21,2 \\
25 \\
15,5 \\
3,4\end{array}$ \\
\hline$m_{\mathrm{g}}\left(\mathrm{kg} \cdot \mathrm{m}^{-2} \cdot \mathrm{jr}^{-1}\right)$ & $\begin{array}{c}5,4 \text { à } 7,1 \\
\text { (non récupérable) }\end{array}$ & $\begin{array}{c}9,4 \\
\text { (non récupérable) }\end{array}$ & 5,8 \\
\hline $\begin{array}{l}\text { C.O.P. }{ }_{\text {th N }} \\
\text { C.O.P.P.th G } \\
\text { C.O.P. } \text { sol N } \\
\text { C.O.P.sol G }\end{array}$ & $\begin{array}{l}0,292 \\
0,277 \\
0,095 \\
0,090\end{array}$ & $\begin{array}{c}<0,33 \\
<- \\
<0,096 \\
-\end{array}$ & $\begin{array}{l}0,335 \\
0,253 \\
0,129 \\
0,097\end{array}$ \\
\hline $\begin{array}{l}S \text { capteur } \\
\text { totale }\left(\mathrm{m}^{2}\right) \\
\eta \text { capteur } \\
\text { Condenseur }\left(\mathrm{m}^{2}\right) \\
\text { Evaporateur }\left(\mathrm{m}^{2}\right)\end{array}$ & $\begin{array}{c}0,8 \\
(1 \text { capteur) } \\
0,327 \\
\text { air }(10) \\
0,65\end{array}$ & $\begin{array}{c}20,3 \\
(24 \text { capteurs) } \\
0,293 \\
\text { eau }(1,7) \\
9\end{array}$ & $\begin{array}{c}6 \\
\text { (4 capteurs) } \\
0,385 \\
\text { air }(24) \\
2,5\end{array}$ \\
\hline
\end{tabular}


relativement basses...) mais leurs inconvénients majeurs sont leurs faibles densités énergétiques et le fait que la chaleur sensible soit très importante vis-àvis de la chaleur d'adsorption.

2. Comparaison des choix et performances des réfrigérateurs basés sur l'utilisation d'une réaction solidegaz.

2.1 LA RÉACTION CHIMIQUE ENTRE UN SOLIDE ET UN GAZ. PRINCIPE DE FONCTIONNEMENT. - La réfrigération basée sur l'utilisation d'une réaction chimique entre un solide et un gaz a été très étudiée, en particulier dans le cadre d'une utilisation de l'énergie solaire. Ce système est particulièrement bien adapté du fait de son intermittence (procédé discontinu) à l'utilisation de l'énergie solaire.

De nombreux couples solide-gaz ont été étudiés mais souvent en vain car de nombreux problèmes ont été rencontrés principalement lors de la mise en œuvre de ces solides (déformations des réacteurs dues au gonflement du sel lors de la première absorption...).

Les réactions solide-gaz présentent par rapport aux systèmes présentés précédemment de nombreux avantages : des densités énergétiques importantes, donc un encombrement plus faible pour une puissance donnée et des puissances importantes qui peuvent être modulées par le contrôle des écarts à l'équilibre thermodynamique.

Nous allons tout d'abord rappeler brièvement le fonctionnement de telles pompes à chaleur chimiques.

Les équilibres mis en jeu dans un tel type de pompe sont :

$$
\begin{aligned}
\langle\mathrm{MX}, n \mathrm{G}\rangle+(p \mathrm{G}) & \rightleftarrows\langle\mathrm{MX},(n+p) \mathrm{G}\rangle \\
{[p \mathrm{G}] } & +\Delta H_{1} \\
& \rightleftarrows(p \mathrm{G})
\end{aligned}
$$

où $\langle\mathrm{MX}, n \mathrm{G}\rangle$ et $\langle\mathrm{MX},(n+p) \mathrm{G}\rangle$ sont deux solides.

Le système est monovariant à l'équilibre, ce qui, dans le diagramme $\operatorname{Ln} P=f(1 / T)$, donne des droites d'équilibre, dans un domaine de température fixé (Fig. 2). Lors de la phase haute pression (désorption-condensation) la quantité de chaleur $\Delta H_{1}$ est fournie à $T_{\mathrm{d}}$ par une source chaude (capteur solaire). Simultanément, la quantité de chaleur $\Delta H_{2}$ est délivrée à $T_{\mathrm{c}}$ et est évacuée, par exemple, par le milieu ambiant (condenseur à air, convection naturelle).

Dans l'étape basse pression (évaporation-absorption), la quantité de chaleur $\Delta H_{2}$ est prélevée au milieu à réfrigérer, à $T_{\mathrm{e}}$, et $\Delta H_{1}$ est délivrée à $T_{\mathrm{a}}$ lors de l'absorption du gaz. $T_{\mathrm{c}}$ et $T_{\mathrm{a}}$ sont souvent proches, notamment lorsque le fluide caloporteur est le même.

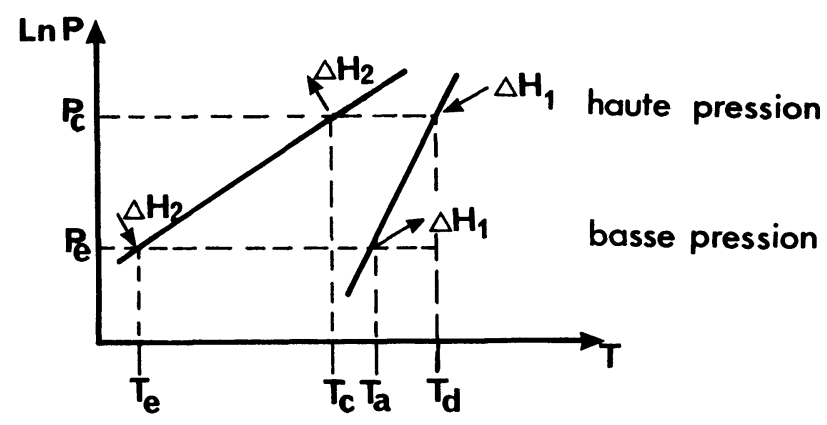

Fig. 2. - Principe de fonctionnement d'une pompe à chaleur chimique en mode réfrigération.

[Functional principle of a chemical heat pump for refrigeration.]

Nous allons présenter une comparaison des différents systèmes de réfrigération utilisant une réaction solide-gaz. Parmi les nombreux auteurs ayant travaillé sur ce sujet, nous citerons plus particulièrement :

$\begin{array}{ll}\text { - Buffington } & 1933 \\ \text { - Andrews } & 1955 \\ \text { - Bonauguri } & 1969 \\ \text { - Muradov-Shadyev } & 1971-72 \\ \text { - Fléchon } & 1977-84 \\ \text { - Worsoe-Schmidt } & 1977-85 \\ \text { - Klvana-Parent } & 1981-84 \\ \text { - Iloeje } & 1985 \\ \text { - Pilatowsky } & 1978 \\ \text { - Roca } & 1983\end{array}$

2.2 CHOIX DU COUPLE SOLIDE-GAZ ET MISE EN GEUVRE DU SOLIDE. - Ce choix repose principalement, dans le cas de la réfrigération solaire, sur les conditions thermodynamiques qui seront imposées au système pendant son fonctionnement. C'est la position de la droite d'équilibre solide-gaz vis-à-vis de celle du gaz-liquide, dans le digramme $\operatorname{Ln} P=$ $f(1 / T)$ qui imposera un couple.

D'autres critères de sélection interviennent ensuite tels que la stabilité chimique, la toxicité, la corrosion, la reproductibilité des réactions de synthèse et de décomposition, la puissance du système...

Pour les différents couples rencontrés dans cette étude, le tableau IV donne les températures d'équilibre minimales dans le cas de la désorption et maximales dans le cas de l'absorption, pour des températures de condensation de 30 et $40^{\circ} \mathrm{C}$, et pour des températures d'évaporation de 0 et $-10^{\circ} \mathrm{C}$. Il faut noter aussi que pour obtenir une vitesse de réaction non négligeable, il faut prendre un écart à l'équilibre de l'ordre de $10^{\circ}$.

Dans le cas de l'utilisation de capteurs solaires plans, à surface non sélective, il apparaît donc difficile d'atteindre avec un rendement acceptable 
Tableau IV. - Températures d'équilibre correspondant aux pressions données pour différents couples solidegaz.

[Equilibrium temperatures corresponding to the given pressures for different solid-gas pairs.]

$T$ cond. $=30^{\circ} \mathrm{C} \quad T$ cond. $=40^{\circ} \mathrm{C} T$ évap. $=-10^{\circ} \mathrm{C} \quad T$ évap. $=0{ }^{\circ} \mathrm{C}$

\begin{tabular}{|c|c|c|c|c|}
\hline \multicolumn{5}{|l|}{$\mathrm{SrCl}_{2} / \mathbf{N H}_{3}$ (éq. 1/8) } \\
\hline$P\left(\mathrm{NH}_{3}\right)$ (bar) & 11 & 15 & 2,7 & 4 \\
\hline$T$ dés. mini $\left({ }^{\circ} \mathrm{C}\right)$ & 92 & 100 & & \\
\hline$T$ abs. maxi $\left({ }^{\circ} \mathrm{C}\right)$ & & & 58 & 66 \\
\hline \multicolumn{5}{|l|}{$\mathrm{CaCl}_{2} / \mathrm{NH}_{3}$} \\
\hline$P\left(\mathrm{NH}_{3}\right)$ (bar) & 11 & 15 & 2,7 & 4 \\
\hline$T$ dés. mini $\left({ }^{\circ} \mathrm{C}\right)($ éq. $2 / 4)$ & 100 & 110 & & \\
\hline$T$ abs. maxi $\left({ }^{\circ} \mathrm{C}\right)($ éq. $4 / 8)$ & & & 50 & 58 \\
\hline \multicolumn{5}{|l|}{$\mathrm{CaCl}_{2} / \mathbf{C H}_{3} \mathbf{N H}_{2}$} \\
\hline$P\left(\mathrm{CH}_{3} \mathrm{NH}_{2}\right)$ (bar) & 4 & 5,5 & 0,85 & 1,3 \\
\hline$T$ dés. mini $\left({ }^{\circ} \mathrm{C}\right)($ éq. $2 / 4)$ & 55 & 60 & & \\
\hline$T$ abs. $\operatorname{maxi}\left({ }^{\circ} \mathrm{C}\right)($ éq. $4 / 6)$ & & & 23 & 30 \\
\hline \multicolumn{5}{|l|}{$\mathrm{LiCl} / \mathrm{CH}_{3} \mathbf{N H}_{2}$ (éq. $2 / 3$ ) } \\
\hline$P\left(\mathrm{CH}_{3} \mathrm{NH}_{2}\right)$ (bar) & 4 & 5,5 & 0,85 & 1,3 \\
\hline$T$ dés. $\operatorname{mini}\left({ }^{\circ} \mathrm{C}\right)$ & 68 & 76 & & \\
\hline$T$ abs. maxi $\left({ }^{\circ} \mathrm{C}\right)$ & & & 35 & 43 \\
\hline
\end{tabular}

les températures hautes nécessaires à la désorption, pour les couples $\mathrm{SrCl}_{2} / \mathrm{NH}_{3}$ et $\mathrm{CaCl}_{2} / \mathrm{NH}_{3}$.

Pour le couple $\mathrm{CaCl}_{2} / \mathrm{CH}_{3} \mathrm{NH}_{2}$, les températures minimales nécessaires pour la décomposition du solide peuvent être atteintes à partir de capteurs plans simples. Par contre, il apparait impossible de produire de la glace à $0{ }^{\circ} \mathrm{C}$, pour ce système, avec une température nocturne de $30^{\circ} \mathrm{C}$, qui correspond précisément à la température d'équilibre du composé $\mathrm{Ca}\left(\mathrm{CH}_{3} \mathrm{NH}_{2}\right)_{2} \mathrm{Cl}_{2}$, à cette pression. Ce système développé par Pilatowsky [11] et Roca [12] a montré son incapacité à produire de la glace, dans de telles conditions de température. Il est plus particulièrement adapté à la climatisation.

Le couple $\mathrm{LiCl} / \mathrm{CH}_{3} \mathrm{NH}_{2}$, cité dans la littérature, n'a jamais été mis en ouvre, mais il apparaît totalement adapté à la réfrigération solaire. La température maximale requise étant de $76^{\circ} \mathrm{C}$, l'utilisation des capteurs solaires plans simples est alors possible. C'est pour ces raisons que nous l'avons choisi.

La mise en œuvre du solide réactionnel a toujours posé de nombreux problèmes, principalement dus au gonflement du solide lors de la première synthèse et à la mauvaise conductivité thermique des solides mis en œuvre dans des lits fixes.

Les auteurs ont cherché à améliorer les transferts de masse par l'adjonction de liants $\left(\mathrm{LiNO}_{3}\right.$ [13-17], ciment de Portland [18, 19, 24], charbon [20], $\mathrm{CaSO}_{4}$ [21]) mais ceux-ci sont de mauvais conducteurs thermiques.

Aussi, après que soit mis en évidence le fait que les transferts de chaleur limitaient les performances du système, des liants conducteurs ont été ajoutés, en particulier des copeaux de métaux tels que la laine d'acier $[22,23]$ ou la mousse d'aluminium. Dans ce cas, la conductivité thermique de ces liants est très élevée, de l'ordre de 500 à 1000 fois celle du sel, ce qui pourtant n'entraîne pas une telle augmentation de la conductivité du mélange dans le lit fixe. Selon le taux de liant, la conductivité effective semble multipliée par un facteur 10 tout au plus.

Pour éviter les déformations d'ailettes dues au gonflement du solide, Buffington [13] a ajouté $5 \%$ de $\mathrm{LiNO}_{3}$ au sel anhydre $\mathrm{SrCl}_{2}, \mathrm{LiNO}_{3}$ devenant liquide lors de l'absorption du gaz $\mathrm{NH}_{3}$. Ce procédé a permis d'éviter de trop fortes contraintes mécaniques. Worsoe-Schmidt [24] qui a essayé ce liant avec $\mathrm{CaCl}_{2}$ constate que ce mélange liquide très visqueux pose des problèmes de diffusion du gaz dans le milieu réactionnel, ce qui entraîne une diminution du taux de conversion final de la réaction. Rappelons que le couple $\mathrm{SrCl}_{2} / \mathrm{NH}_{3}$ a été mis en œuvre, à l'échelle industrielle, par la Faraday Refrigeration $\mathrm{C}^{\circ}$, aux Etats-Unis, dans un réfrigérateur ménager, mais en l'absence de tout liant.

Bonauguri [20] a utilisé un mélange contenant des proportions massiques de $31,2 \%$ de charbon pur sous forme de petites boules, et de $68,8 \%$ de $\mathrm{CaCl}_{2}$, dans le but d'éviter le colmatage du sel et de faciliter le transfert de gaz lors de l'absorption. Elle n'a constaté aucune amélioration ni de l'homogénéité du sel, ni de la vitesse de réaction lors de la synthèse.

Worsoe-Schmidt $[18,19,24]$ a utilisé un mélange de $15 \%$ de ciment de Portland et l'auteur a constaté 
une augmentation sensible de la vitesse de réaction ainsi qu'une amélioration du taux de conversion final. Par contre, ce procédé ne semble pas éliminer les problèmes liés aux contraintes mécaniques et a amené l'auteur à concevoir un nouveau type de réacteur à partir du choix d'un autre liant $\mathrm{LiNO}_{3}$ mentionné précédemment.

Toujours dans le but de réduire les effets dus au gonflement du sel, Iloeje [21] a successivement utilisé le ciment de Portland, puis un mélange à $20 \%$ de $\mathrm{CaSO}_{4}$. Avec ce dernier, il observe un meilleur cyclage mais il ne parle pas d'un effet quelconque sur la vitesse de réaction.

Dans le but d'améliorer les transferts de chaleur, Klvana et Parent $[22,23]$ ont utilisé des couches de laine d'acier en alternance avec des couches de sel. Ceci leur a permis d'une part d'améliorer la conductivité apparente du lit réactionnel mais aussi d'assurer un support mécanique poreux pour le sel. Ces auteurs affirment observer, cycle après cycle, une augmentation du volume occupé par le sel. Ceci n'est, à notre avis, pas compatible avec le fait que les produits de la réaction sont des composés définis, à volumes molaires déterminés. Une telle structure de réacteur leur permet d'obtenir des réactions complètes d'une durée de $18 \mathrm{~h}$ aussi bien en synthèse qu'en décomposition pour des écarts à l'équilibre faibles (de l'ordre de 3 à $5^{\circ}$ ).

Dès 1982, au laboratoire, Heydari [25] a mis en évidence le rôle très important d'un liant graphité sur la conversion du solide réactionnel. Ce liant, dont on a fait varier le pourcentage massique, au cours des essais, de 5 à $50 \%$, a permis de multiplier par un facteur 8, dans le cas du couple $\mathrm{CaCl}_{2} / \mathrm{CH}_{3} \mathrm{NH}_{2}$ et au-delà pour d'autres couples $(20$ pour $\mathrm{ZnCl}_{2} / \mathrm{CH}_{3} \mathrm{NH}_{2}$ ), la vitesse de réaction.

Ce liant a été utilisé par Roca [12] en proportion de $20 \%$ lors d'expérimentations sur une maquette de réfrigérateur solaire. Nous avons utilisé ce liant avec $\mathrm{LiCl}$ en proportion de $40 \%$ et nous verrons plus loin les résultats obtenus.

Le cyclage des solides mis en jeu dans les pompes à chaleur chimiques est un critère très important dans le cadre d'une réalisation à l'échelle industrielle. Lorsqu'aucun liant n'est utilisé et dans le cas des forts tassements [13], le cyclage se traduit, après quelques dizaines de cycles par un colmatage du sel : celui-ci forme des agrégats et le taux de conversion diminue alors fortement.

Les cyclages réalisés sur des maquettes sont ceux de Worsoe-Schmidt $[17,24]$ sur le couple $\mathrm{CaCl}_{2} / \mathrm{NH}_{3}$ avec deux types de liant : $15 \%$ ciment de Portland puis $\mathrm{LiNO}_{3}$ (le pourcentage n'est pas précisé) et de Heydari [25] sur le couple $\mathrm{CaCl}_{2} / \mathrm{CH}_{3} \mathrm{NH}_{2}$ avec $20 \%$ de liant graphité.

Le cyclage réalisé par Heydari a montré qu'il n'y avait pas d'altération du solide, ni de changement de la vitesse de réaction : 170 cycles ont été réalisés.
Celui de Worsoe-Schmidt et al. dans le cas de $\left(\mathrm{CaCl}_{2}+15 \%\right.$ de ciment de Portland) [24] ne s'est pas avéré satisfaisant. Ils ont constaté des diminutions des performances du réacteur qu'ils attribuent au déplacement du tube distributeur de gaz sans évoquer les éventuelles modifications physico-chimiques.

Plus récemment, ces auteurs, après avoir conçu un nouveau réacteur solide-gaz, ont testé le mélange $\left(\mathrm{CaCl}_{2}+\mathrm{LiNO}_{3}\right)$ et ont réalisé 670 cycles [17]. Après avoir découpé le réacteur en plusieurs endroits, ils ont constaté que la structure poreuse était restée homogène.

Le tassement du sel dans le réacteur est aussi un facteur très important tant au niveau de la puissance $\mathrm{du}$ système (puissance de la réaction solide-gaz et donc puissance lors de la production de froid) que de son encombrement et donc de son coût. Il en est de même pour la densité énergétique volumique liée au tassement et définie de la façon suivante :

$$
D_{\mathrm{E}}=X \cdot p \cdot \Delta H \cdot\left(m_{\mathrm{s}} / M_{\mathrm{s}} \cdot V\right)
$$

avec $X$ taux d'avancement de la réaction, $p$ nombre de moles échangées au cours de la réaction, $\Delta H$ variation d'enthalpie de la réaction solide-gaz, $m_{\mathrm{s}}$ masse de sel introduite dans le réacteur, $M_{\mathrm{s}}$ masse molaire du sel et $V$ volume utile du réacteur; le rapport $m_{\mathrm{s}} / M_{\mathrm{s}} V$ représentant le tassement du solide dans le réacteur, et s'exprimant en moles de sel par $\mathrm{m}^{3}$ de réacteur.

La notion de densité énergétique est très importante. Elle permet de situer l'importance des volumes mis en jeu pour une puissance donnée. Dans le cas de capteurs solaires, où le volume est limité (pour ne pas avoir des systèmes trop encombrants), le choix du solide se portera sur un couple à grande densité énergétique.

Mais ce facteur n'est pas le seul à devoir être pris en compte. Mauran [35] a en effet démontré que pour tous les couples solide-gaz, il y avait un optimum de tassement, au-delà duquel la réaction n'est plus complète. Dans le cas des couples étudiés, les valeurs des tassements et des densités énergétiques maximales sont données dans le tableau $\mathrm{V}$.

Remarque : nous voyons donc que, dans le cas du couple $\mathrm{CaCl}_{2} / \mathrm{NH}_{3}$, pour que la seconde réaction d'absorption ait lieu totalement, il ne faut pas dépasser le tassement de 4830 moles de sel par $\mathrm{m}^{3}$ de réacteur.

Le tableau $\mathrm{V}$ nous donne aussi les valeurs des tassements utilisés par les différents auteurs. Dans presque tous les cas, nous remarquons que le compactage utilisé est toujours inférieur à l'optimum donné par Mauran. La réaction complète peut donc avoir lieu. La plupart des auteurs ont choisi un tassement moyen compris entre 2000 et 2700 moles 
Tableau V. - Caractéristiques des mélanges réactionnels utilisés par les auteurs.

[Characteristics of the reactive medium used by the authors.]

\begin{tabular}{|c|c|c|c|c|c|c|c|}
\hline $\begin{array}{l}\text { Couples et } \\
\text { équilibres }\end{array}$ & Auteurs & Liant (\% masse) & Cyclage & $\begin{array}{l}\text { Tassement } \\
\text { mol. sel } / \mathrm{m}^{3}\end{array}$ & $\begin{array}{c}\text { Densité } \\
\text { énergétique } \\
\mathrm{MJ} / \mathrm{m}^{3} \\
\text { (X exp. max) }\end{array}$ & $\operatorname{Tas}^{t} \max$ & $D_{\mathrm{E}} \max$ \\
\hline $\begin{array}{c}\mathrm{SrCl}_{2} / \mathrm{NH}_{3} \\
\text { (é. } 1 / 8)\end{array}$ & Buffington & $5 \% \mathrm{LiNO}_{3}$ & mauvais & 5070 & $650(0,42)$ & 4490 & 1373 \\
\hline \multirow{8}{*}{$\begin{array}{l}\mathrm{CaCl}_{2} / \mathrm{NH}_{3} \\
\text { (éq. } 2 / 4) \\
\text { (éq. } 4 / 8)\end{array}$} & Andrews & 0 & 2 ans & 2165 & $<550$ & \multirow{8}{*}{$\begin{array}{l}7830 \\
4830\end{array}$} & \multirow{8}{*}{$\begin{array}{l}665 \\
821\end{array}$} \\
\hline & Bonauguri & $\begin{array}{c}31,2 \% \text { charbon } \\
\text { pas d'effet } \\
\text { observé }\end{array}$ & $\begin{array}{c}\text { mauvais } \\
30 \text { cycles } \rightarrow \\
\text { blocs }\end{array}$ & - & - & & \\
\hline & $\begin{array}{l}\text { Muradov- } \\
\text { Shadyev }\end{array}$ & 0 & - & 1920 & $<490$ & & \\
\hline & Fléchon & 0 & - & 1992 & - & & \\
\hline & Klvana-Parent & $\begin{array}{l}47 \% \text { en } \\
\text { volume de } \\
\text { laine d'acier }\end{array}$ & - & 2480 & $337(0,80)$ & & \\
\hline & $\begin{array}{l}\text { Worsoe-Schmidt } \\
\text { prototype I }\end{array}$ & $\begin{array}{l}15 \% \text { ciment } \\
\text { de Portland }\end{array}$ & $\begin{array}{l}232 \text { cycles } \\
(6 \text { mois })\end{array}$ & 2100 & $482 \quad(0,90)$ & & \\
\hline & $\begin{array}{l}\text { Worsoe-Schmidt } \\
\text { prototype II }\end{array}$ & $\mathrm{LiNO}_{3}$ & 670 cycles & 2460 & $<627$ & & \\
\hline & Iloeje & $20 \% \mathrm{CaSO}_{4}$ & non & 2772 & $127(0,18)$ & & \\
\hline \multirow{2}{*}{$\begin{array}{l}\mathrm{CaCl}_{2} / \mathrm{CH}_{3} \mathrm{NH}_{2} \\
\text { (éq. } 2 / 4 \text { ) } \\
\text { (éq. } 4 / 6)\end{array}$} & Pilatowsky & 0 & 28 cycles & 1461 & $<292$ & \multirow[t]{2}{*}{$\begin{array}{l}\text { (éq. } 2 / 6) \\
3660\end{array}$} & \multirow[t]{2}{*}{730} \\
\hline & Roca & $20 \%$ graphex & $\begin{array}{r}170 \text { cycles } \\
\text { (Heydari) }\end{array}$ & 870 & 174 & & \\
\hline
\end{tabular}

de sel par $\mathrm{m}^{3}$ pour le couple $\mathrm{CaCl}_{2} / \mathrm{NH}_{3}[14-24,26$, 28].

Il faut noter que malgré ces faibles valeurs de tassement, certains auteurs n'ont pas réalisé de réactions complètes. C'est le cas de Iloeje [21] qui obtient des taux d'avancement maxima de 0,18 , cette faible valeur étant due aux limitations par transferts de chaleur et de masse. Seul, Buffington [13] a utilisé un compactage supérieur à celui donné par Mauran. Il est donc évident qu'il ne peut obtenir des réactions complètes et il atteint expérimentalement des taux de réaction maxima de 0,42.

Compte tenu de l'importance du tassement maximum, il est utile d'optimiser cette valeur en vue d'une application pratique, en particulier, dans le cas de machines compactes où il faut cycler le maximum de gaz réfrigérant pour un minimum d'encombrement du réacteur solide-gaz.
2.3 LES RÉACTEURS SOLIDE-GAZ. - La plupart des auteurs ayant réalisé des expérimentations sur des maquettes ou des prototypes de pompe à chaleur (en mode chauffage ou production de froid) ont constaté que les limitations essentielles lors de la mise en cuvre des systèmes sont dues aux transferts de chaleur et de matière.

Dans le cas des réactions solide-gaz, c'est la synthèse ou la décomposition du solide qui limite la puissance globale du système car elle est toujours plus lente que le processus d'évaporation ou de condensation du gaz.

Cette limitation est principalement due à la mauvaise conductivité thermique des sels réactifs mis en œuvre dans des lits fixes. Shadyev et Umarov [29] ont calculé, à partir de profils de température en fonction du rayon dans le milieu réactionnel, la conductivité apparente de ce milieu. Ils obtiennent 
des valeurs qui décroissent de 2,8 à $0,76 \mathrm{~W} \cdot \mathrm{m}^{-1} \cdot \mathrm{K}^{-1}$ au cours de la désorption mais sans apporter de remarques quant à cette différence. Celle-ci peut être reliée à la porosité du milieu : la valeur de $2,8 \mathrm{~W} \cdot \mathrm{m}^{-1} \cdot \mathrm{K}^{-1}$ correspond à la composition $\mathrm{Ca}\left(\mathrm{NH}_{3}\right)_{8} \mathrm{Cl}_{2}$ et celle de $0,76 \mathrm{~W} \cdot \mathrm{m}^{-1} \cdot \mathrm{K}^{-1}$ à un milieu plus poreux, de composition $\mathrm{Ca}\left(\mathrm{NH}_{3}\right)_{4} \mathrm{Cl}_{2}$.

$\mathrm{La}$ remarque précédente ainsi que nos propres études montrent qu'en fait la limitation n'est pas purement due au transfert de chaleur. C'est le couplage de la cinétique chimique et des transferts de chaleur et de masse dans le milieu réactionnel qui limite le phénomène. Mazet [36] a démontré l'existence de ce couplage à partir d'expérimentations sur le couple $\mathrm{CaCl}_{2} / \mathrm{CH}_{3} \mathrm{NH}_{2}$, en phase de désorption. A partir de ces mesures, elle a établi que la distance maximale entre le sel et la paroi de l'échangeur en contact avec celui-ci devait être inférieure à $2 \mathrm{~cm}$ pour que le milieu réactionnel soit quasi isotherme.

Ce résultat est très important en vue de la construction de prototypes. L'échangeur du réacteur solide-gaz d'une pompe à chaleur chimique doit être calculé de façon à respecter cette distance maximale entre le sel et la paroi de l'échangeur.

Dans le cadre de la réfrigération solaire, il est fondamental de tenir compte de ce facteur dans les réacteurs tubulaires où le sel est en contact direct avec la paroi de l'échangeur.

Parmi les auteurs dont les travaux sont présentés, Klvana et Parent [23] ont abordé ce point de façon systématique. Ils se sont penchés sur ce problème à la suite des résultats obtenus sur un premier pilote. Des mesures de température effectuées au niveau de la surface du tube du réacteur solide-gaz et au centre de ce même tube ont donné des écarts de l'ordre de 17 à $30^{\circ}$, pour un réacteur ayant une distance maximale sel-échangeur de $10 \mathrm{~cm}$ environ [22]. Cette non-isothermicité importante entraîne dès la $2^{c}$ heure de synthèse un ralentissement très net de la vitesse. Dans le réacteur, on peut alors distinguer 2 zones. La zone centrale est au départ dans le domaine de désorption $\left(\mathrm{Ca}(\mathrm{NH} 3)_{8} \mathrm{Cl}_{2} \rightarrow\right.$ $\mathrm{Ca}(\mathrm{NH} 3)_{4} \mathrm{Cl}_{2}$ noté $8 \rightarrow 4$ ) et celui de l'absorption $2 \rightarrow 4$. Ceci reste vrai jusqu'au passage de l'équilibre $\left(5^{\mathrm{c}}\right.$ heure). Cette partie du réacteur se situe alors dans le domaine d'absorption $4 \rightarrow 8$. La vitesse d'absorption est donc faible dans cette zone. Quant à la zone située près de l'échangeur (surface), il apparaît qu'elle est toujours dans le domaine d'absorption $4 \rightarrow 8$. C'est donc elle qui réagit avec la plus grande vitesse. Ceci justifie bien le fait que la réaction débute près de l'échangeur et d'autre part que le rayon du réacteur solide-gaz est trop élevé et conduit à de forts gradients de température dans le solide réactionnel.

Les auteurs cités dans cette étude bibliographique ont conçu des réacteurs solide-gaz présentant des similitudes. Ils utilisent le plus souvent des tubes cylindriques parallèles entre eux et reliés à un collecteur ; c'est le cas de Bonauguri, Muradov et Shadyev, Klvana et Parent, Worsoe-Schmidt, Iloeje et Pilatowsky.

Dans ces tubes, le sel est en contact direct avec la paroi et l'arrivée de gaz est la plupart du temps centrale, chaque tube ayant une arrivée de gaz.

Comme nous l'avons précisé précédemment, le couplage vitesse de réaction-transferts a entrainé la détermination d'une distance maximale entre le sel et l'échangeur. Cette distance varie de 1,2 à $5 \mathrm{~cm}$ selon les auteurs.

Dans le tableau VI, nous avons regroupé les différentes caractéristiques de chaque réacteur solide-gaz ainsi que leur mode de chauffage et de refroidissement, et la structure des condenseurs et évaporateurs utilisés.

Le rayonnement par lampes IR et/ou le rayonnement solaire pour la phase de désorption-condensation a été utilisé par Bonauguri, Muradov-Shadyev, Fléchon, Klvana-Parent, Worsoe-Schmidt et Iloeje. Dans les autres cas, un fluide caloporteur est utilisé (Buffington, Andrews, Pilatowsky et Roca).

Les mesures effectuées avec le rayonnement IR artificiel constituent une meilleure simulation du comportement de la machine en ensoleillement réel que celle obtenue par l'intermédiaire d'un fluide caloporteur.

Pour le refroidissement, divers types sont proposés :

- convection naturelle, par exemple, par effet cheminée dans le capteur contrôlée par l'intermédiaire de volets (Bonauguri, Muradov-Shadyev, Fléchon, Klvana-Parent, Worsoe-Schmidt et Iloeje) ;

- convection forcée (ventilation): WorsoeSchmidt et Iloeje ;

- retrait de l'isolation arrière du capteur ou du vitrage (Worsoe-Schmidt, prototype I) ;

- fluide caloporteur (Bonauguri, Andrews, Worsoe-Schmidt, Pilatowsky et Roca).

Le refroidissement se rapprochant le plus de celui utilisable en fonctionnement réel de l'appareil, de façon autonome, est le refroidissement par effet cheminée par ouverture des volets situés en bas et en haut du capteur. L'ouverture de ces volets peut être commandée à partir d'un capteur de pression différentielle ou programmée à partir d'une horloge (lever et coucher du soleil). Seul Worsoe-Schmidt [14] utilise une circulation de liquide de refroidissement R11 par thermosiphon.

2.4 Condenseurs et Évaporateurs. - Les condenseurs et évaporateurs utilisés par les auteurs précités sont constitués le plus souvent de tubes à ailettes pour les premiers et de tubes lisses ou de réservoirs cylindriques pour les seconds.

Les condenseurs sont fréquemment des échan- 
Tableau VI. - Caractéristiques des réacteurs solide-gaz, de leurs modes de chauffage et de refroidissement et de la structure des condenseurs et évaporateurs.

[Characteristics of the solid-gas reactors, of their heating and cooling systems and their condensers and evaporators structures.]

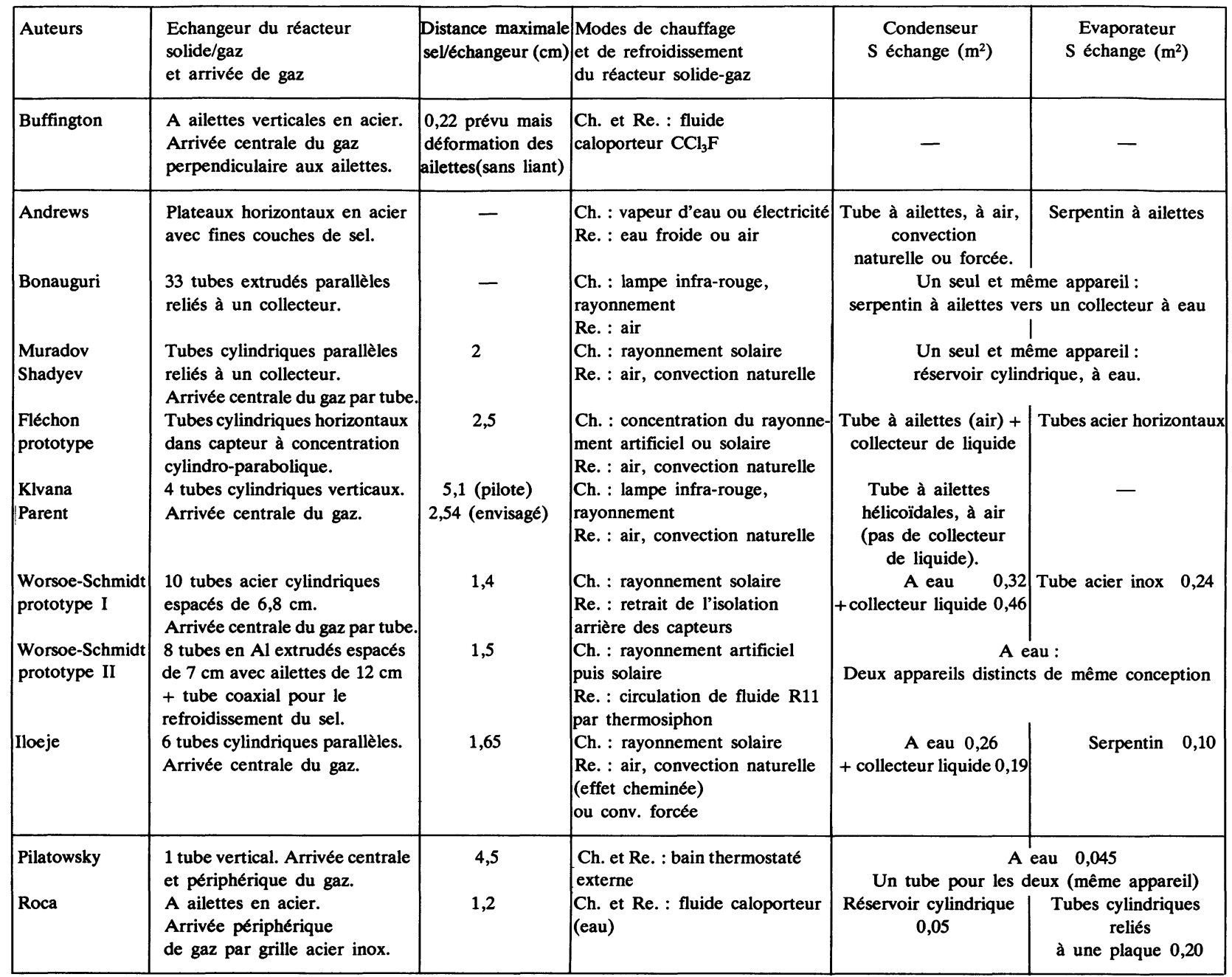

geurs à eau ; c'est le cas de Bonauguri, MuradovShadyev, Worsoe-Schmidt, Iloeje et Pilatowsky.

Dans le cas de la réfrigération solaire, il faut que condenseur et évaporateur soient deux appareils distincts. Si ce n'est pas le cas, la chaleur dégagée lors de la condensation entraîne une fusion de la glace formée pendant la nuit. Dans de nombreux montages, ce défaut de conception est rencontré : Bonauguri et Muradov-Shadyev.

D'autre part, dans la plupart des cas présentés dans le tableau VI, aucune mention n'est faite du calcul des surfaces d'échange. Dans le cas où elles sont données, nous pouvons évaluer la puissance évacuée lors du refroidissement du gaz suivi de sa condensation. Cette puissance est donnée par : $P_{\mathrm{C}}=\left(\left(m \cdot C_{\mathrm{p}_{\mathrm{g}}} \cdot \Delta T\right)+m \cdot L_{\mathrm{e}}\right) / t_{\mathrm{dés}}$ avec $m$ masse de gaz condensé $(\mathrm{kg}), C_{\mathrm{p}_{\mathrm{g}}}$ capacité calorifique massique du gaz $\left(\mathrm{kJ} . \mathrm{kg}^{-1} \cdot \mathrm{K}^{-1}\right), \Delta T$ différence entre la température du gaz à l'entrée du condenseur et la température du liquide à sa sortie (ou dans le collecteur lorsqu'il y en a un), $t_{\text {dés }}$ durée de la désorption (s) et $L_{\mathrm{e}}$ chaleur latente de condensation $\left(\mathrm{J} . \mathrm{kg}^{-1}\right)$.

A partir des valeurs de la puissance libérée lors de la condensation et de la surface d'échange, on obtient les valeurs moyennes de coefficient d'échange pour les condenseurs suivantes :

Worsoe-Schmidt $\quad P_{\mathrm{C}}=403 \mathrm{~W} h_{\mathrm{c}}=8,6 \mathrm{~W} \cdot \mathrm{m}^{-2} \cdot \mathrm{K}^{-1}$

Iloeje

$$
P_{\mathrm{C}}=63,2 \mathrm{~W}
$$$$
2,3 \mathrm{~W} \cdot \mathrm{m}^{-2} \cdot \mathrm{K}^{-1}
$$

Roca

$$
P_{\mathrm{C}}=10,6 \mathrm{~W}
$$$$
7,0 \mathrm{~W} \cdot \mathrm{m}^{-2} \cdot \mathrm{K}^{-1}
$$

La seule remarque que nous puissions faire concerne la faible valeur obtenue par Iloeje qui pourtant utilise un condenseur à eau. 
2.5 MAQUETTES ET PROTOTYPES. - La plupart des auteurs ont effectué leurs expériences sur des bancs d'essai en laboratoire. Les réalisations de prototype sont au nombre de 7 :

- le réfrigérateur Faraday [13];

- le wagon réfrigérant d'Andrews [26, 27] ;

- la machine à glace de Muradov-Shadyev [28] ;

- le réfrigérateur solaire de Fléchon [34] ; 15] ;

- les 2 prototypes I et II de Worsoe-Schmidt [14,

- la machine à glace de Iloeje [21].

En règle générale, le fonctionnement de ces appareils se fait par l'intermédiaire de vannes ce qui ne fait pas apparaître l'automatisme possible de ces systèmes. Quelques systèmes utilisent des clapets anti-retour ou tout autre moyen permettant cet automatisme comme c'est le cas pour :

- le wagon d'Andrews : la vapeur d'eau actionne les vannes; de plus, une vanne à flotteur permet de stopper le débit de vapeur quand suffisamment de gaz est condensé ;

- le prototype II de machine à glace développé par Worsoe-Schmidt : il est prévu dans la suite qu'une vanne thermostatique impose au liquide de refroidissement (R11) de ne circuler dans le réacteur-solide-gaz qu'en phase d'absorption.

Le fonctionnement des prototypes du tableau VI est semblable, aussi allons nous préciser tout d'abord le protocole expérimental classique suivi en signalant les variantes éventuelles.

Au départ, pendant le jour, lorsque le capteur contenant le réacteur solide-gaz est chauffé par le rayonnement solaire (ou simulé par des lampes IR), le réacteur est en communication avec le condenseur. Pendant une courte période $(1$ à $2 \mathrm{~h}$ selon les conditions), le sel dans le réacteur s'échauffe sans qu'il y ait réaction chimique. Dès que les conditions de température et de pression sont telles que la désorption est possible, celle-ci débute et entraîne une baisse de la température du solide. La réaction se poursuit tant que le chauffage du réacteur est maintenu (variable de 5 à $10 \mathrm{~h}$ ). Dès l'arrêt du rayonnement incident, la température du réacteur et, avec un certain décalage, celle du sel, commencent à diminuer. Ceci se fait par l'ouverture de volets situés en haut et en bas du capteur ou par le retrait de l'isolation arrière; ces différents moyens de refroidissement ayant été précisés précédemment (cf. Tab. VI).

Le liquide condensé est stocké dans un collecteur ou dans le condenseur lui-même. Il est alors transféré dans l'évaporateur. Dans le cas où condenseur et évaporateur sont un seul et même appareil, ce transfert n'a pas lieu. Dès que la température du sel dans le réacteur est suffisamment basse, la phase d'absorption-évaporation débute et se poursuit jusqu'au lendemain matin.
Dans certains cas (Iloeje par ex.), l'absorbeur et l'évaporateur ne sont mis en communication que lorsque les conditions d'écart à l'équilibre sont réalisées. La vitesse de réaction est alors, au début, rapide et on assiste à une forte montée des températures du solide dans le capteur, due à l'exothermicité de l'absorption. Les temps de mise en température varient de 3,6 à $4,8 \mathrm{~h}$ avant que l'absorption ne débute.

La plupart des auteurs imposent, dans le protocole expérimental, des mises en température plus progressives. Ce fonctionnement traduit mieux l'utilisation, en site réel, du prototype.

Nous avons vu précédemment que les conditions de fonctionnement dépendent principalement des écarts à l'équilibre. Mais, étant donné que le fonctionnement des systèmes se fait en régime dynamique, les écarts à l'équilibre varient au cours du temps. Ainsi, lors de la phase de condensation, la pression évolue, en particulier dans le cas des condenseurs à air (la pression de condensation est plus stable dans le cas des condenseurs à eau car la température du fluide refroidisseur est plus constante dans le temps). Il en est de même pour la phase d'évaporation tant que l'eau n'a pas atteint la température de $0{ }^{\circ} \mathrm{C}$.

Dans l'ensemble des résultats présentés par les différents auteurs, il apparaît que les écarts à l'équilibre sont très faibles (de l'ordre de 3 à $8^{\circ}$ au maximum).

Les courbes de variation des températures en fonction du temps ainsi que celles donnant les taux d'avancement montrent que les réactions de désorption et d'absorption débutent pour de très faibles écarts, de l'ordre de quelques degrés ; il en résulte une vitesse faible.

En conclusion, nous pouvons retenir que la structure d'échangeur du réacteur solide-gaz la plus utilisée est celle comportant des tubes cylindriques avec arrivée de gaz centrale. Ce système permet d'avoir de faibles valeurs de la distance maximale sel-échangeur grâce à des diamètres choisis petits, mais cela entraîne une augmentation de la masse de l'absorbeur, ce qui représente en moyenne une consommation d'énergie sous forme de chaleur sensible de l'ordre du quart de la chaleur nécessaire pour effectuer la désorption.

2.6 RÉSULTATS SUR LES CINÉTIQUES, LES PUISSANCES ET LES C.O.P. - Dans les systèmes de réfrigération solaire dont le fonctionnement s'effectue le plus souvent en régime transitoire, il est impossible de relier la cinétique de réaction à des paramètres $(T, P)$ fixes. Dans de tels systèmes, en effet, la pression de condensation ainsi que la température du sel varient constamment durant la phase de chauffage-désorption, aussi il est très difficile de déterminer un écart à l'équilibre et donc des condi- 
tions thermodynamiques de fonctionnement précises. Il en est de même pour la phase de refroidissement-absorption car l'évaporation ne se fait pas à température constante.

Dans ces systèmes, l'examen simultané des courbes de température et de taux d'avancement en fonction du temps montre que le sel est toujours à une température très proche de celle correspondant à l'équilibre solide-gaz, l'écart étant de l'ordre de quelques degrés seulement. Il atteint, très rarement, $10^{\circ}$ en fin de réaction.

Dans le tableau VII sont indiquées les performances des différents systèmes de réfrigération solaire réalisés.

La puissance extraite à l'évaporateur est calculée à partir de la masse de gaz évaporée lors de l'absorption, de façon globale, c'est-à-dire entre le début $t=0$ et la fin de l'absorption ( $t_{\mathrm{f}}$ variant de $1 \mathrm{~h}$ [13] à $20 \mathrm{~h}$ [20]). Elle est exprimée en $\mathrm{W}$ par $\mathrm{kg}$ de sel et pour un cycle équivalant à une désorption suivie d'une absorption.

Nous remarquons, que, dans de tels systèmes, cette puissance est faible, de l'ordre de la dizaine de W. Ceci est lié en partie, au faible écart à l'équilibre et au tassement du solide dans le réacteur, deux paramètres qui contrôlent l'absorption du gaz par le sel.
Ce tableau comporte les temps de réaction d'absorption et de désorption observés expérimentalement, exprimés en heures.

Dans le cas de Buffington et Andrews, ces valeurs ne sont données qu'à titre indicatif. On ne peut pas expliquer pourquoi l'absorption est plus rapide que la désorption dans le cas d'Andrews [27] étant donné que l'on ne possède aucun renseignement quant au fonctionnement du système.

Pour les autres auteurs, la durée de l'absorption est toujours supérieure à celle de la désorption, cela étant logique pour un fonctionnement réel où les heures d'ensoleillement sont moins nombreuses que les autres.

Dans le cas d'Iloeje [21], il faut ajouter aux temps de réaction d'absorption propre, les temps correspondant au refroidissement ce qui conduit à des valeurs comprises entre 8,5 et $9,25 \mathrm{~h}$.

Dans la plupart des cas rencontrés, on ne connaît pas précisément les cinétiques de réaction, les auteurs ne précisant pas suffisamment, les conditions de pression et température.

Le tableau VII indique aussi la nature de la production de froid (air froid ou glace), la masse de glace formée et le rapport masse de glace sur masse de solide.

La comparaison des performances obtenues par

Tableau VII. - Performances des réfrigérateurs présentés par les auteurs.

[Performances of the refrigerators presented by the authors.]

\begin{tabular}{|c|c|c|c|c|c|c|c|c|c|}
\hline \multirow[t]{2}{*}{ Auteurs } & \multirow{2}{*}{$\begin{array}{c}\text { Puissance } \\
\text { extraite à } \\
\text { l'évaporateur/cycle } \\
\text { (W/kg sel) }\end{array}$} & \multirow{2}{*}{$\begin{array}{l}t_{\text {abs. }}(\mathrm{h}) \\
t_{\text {dés. }}(\mathrm{h})\end{array}$} & \multirow{2}{*}{$\begin{array}{c}\text { Nature de la } \\
\text { production } \\
\text { de froid } \\
\left(m_{\mathrm{g}} \text { en } \mathrm{kg} \cdot \mathrm{m}^{-2} \cdot \mathrm{j}^{-1}\right)\end{array}$} & \multirow{2}{*}{$\frac{\text { Masse de glace }}{\text { Masse de solide }}$} & \multicolumn{2}{|c|}{ C.O.P. th } & \multicolumn{2}{|c|}{ C.O.P. sol } & \multirow[t]{2}{*}{$\eta$ capteur } \\
\hline & & & & & E & G & $\mathbf{E}$ & $\mathbf{G}$ & \\
\hline Buffington & $\begin{array}{c}60 \text { (12 cycles/j) } \\
5(1 \text { cycle })\end{array}$ & $\begin{array}{l}1 \\
1\end{array}$ & $\begin{array}{c}\text { glace fondante } \\
\left(0^{\circ} \mathrm{C}\right) \\
(45 \text { sur } 12 \text { cycles }) \\
(3,7 \text { par cycle })\end{array}$ & 1,02 & \begin{tabular}{|l|}
$\ll 0,534$ \\
(sans chaleu
\end{tabular} & $\begin{array}{l}<0,432 \\
\text { (r sensible) }\end{array}$ & - & - & - \\
\hline Andrews & $\begin{array}{c}38,6(6 \text { cycles } / j) \\
6,4(1 \text { cycle })\end{array}$ & $\begin{array}{l}0,67 \\
3\end{array}$ & $\begin{array}{l}\text { air froid } \\
(0)\end{array}$ & 0 & - & 0 & - & 0 & - \\
\hline Bonauguri & de 6,3 à 8,3 & $\begin{array}{c}15-20 \\
4-7\end{array}$ & $\begin{array}{l}\text { glace } \\
(1,3)\end{array}$ & 0,94 & $\mid \begin{array}{l}<0,20 \\
\text { (sans chaleur }\end{array}$ & $\begin{array}{l}<0,133 \\
\text { r sensible) }\end{array}$ & 0,020 & 0,015 & $>0,10$ \\
\hline $\begin{array}{l}\text { Muradov- } \\
\text { Shadyev }\end{array}$ & de 9,8 à 10,3 & $\begin{array}{l}14 \\
10\end{array}$ & $\begin{array}{l}\text { glace } \\
(4)\end{array}$ & 1,43 & $\mid$\begin{tabular}{c|}
$<0,382 \quad \mid$ \\
(sans chaleur
\end{tabular} & $\begin{array}{l}<0,333 \\
r \text { sensible) }\end{array}$ & 0,071 & 0,062 & $>0,186$ \\
\hline $\begin{array}{l}\text { Fléchon } \\
\text { prototype }\end{array}$ & 5,3 & $\begin{array}{r}18 \\
6\end{array}$ & $\begin{array}{l}\text { glace } \\
(1,4)\end{array}$ & 0,67 & $\mid \begin{array}{l}\ll 0,548 \mid \\
\text { (sans chaleur }\end{array}$ & $\begin{array}{l}<0,067 \\
\text { r sensible) }\end{array}$ & $\begin{array}{l}0,095 \\
\quad(\mathrm{sem}\end{array}$ & $\begin{array}{l}0,012 \\
\text { i-concen }\end{array}$ & $\mid>0,173$ \\
\hline Klvana-Parent & - & 15 & $\begin{array}{c}\text { glace } \\
(-)\end{array}$ & & - & - & - & - & - \\
\hline $\begin{array}{l}\text { Worsoe-Schmidt } \\
\text { prototype I }\end{array}$ & 19,2 & $\begin{array}{r}14 \\
7\end{array}$ & $\begin{array}{l}\text { glace } \\
\left(6\left(^{*}\right)\right)\end{array}$ & 2,67 & 0,396 & 0,327 & 0,119 & 0,098 & 0,300 \\
\hline $\begin{array}{l}\text { Worsoe-Schmidt } \\
\text { prototype II }\end{array}$ & $\begin{array}{c}\text { de } 20 \text { à } 24 \\
\text { (sans pertes) }\end{array}$ & $\begin{array}{l}12-15,7 \\
7,3-8,3\end{array}$ & $\begin{array}{c}\text { glace } \\
\left(2,6\left(^{*}\right)\right)\end{array}$ & 0,83 & 0,317 & 0,085 & 0,134 & 0,036 & 0,423 \\
\hline Iloeje & de 10,2 à 17,3 & $\begin{array}{l}3,75-5,67 \\
6,75-6,80\end{array}$ & $\begin{array}{ll} & \text { glace } \\
(0,41) & (0,7 \text { maxi. })\end{array}$ & 0,096 & 0,267 & 0,044 & 0,063 & 0,010 & 0,236 \\
\hline Roca & 28 & $\begin{array}{c}8 \\
10\end{array}$ & $\begin{array}{l}\text { eau froide } \\
(0)\end{array}$ & 0 & 0,360 & 0 & - & 0 & - \\
\hline Balat & de 12 à 14 & $\begin{array}{r}5 \\
15\end{array}$ & $\begin{array}{l}\text { glace } \\
(2,5)\end{array}$ & 1 & 0,322 & 0,161 & 0,107 & 0,053 & 0,33 \\
\hline
\end{tabular}

$\left(^{*}\right) T$ de l'eau initiale $=0^{\circ} \mathrm{C}$. 
les divers auteurs est difficile dans la mesure où, pour fabriquer de la glace, ils ne partent pas des mêmes conditions initiales. C'est ainsi que MuradovShadyev [28] obtient $4 \mathrm{~kg} / \mathrm{m}^{2}$. jour en ayant mis au départ de l'eau à $20^{\circ} \mathrm{C}$ alors que Worsoe-Schmidt [19] indique une valeur de $6 \mathrm{~kg} / \mathrm{m}^{2}$. jour, mais à partir d'eau à $0^{\circ} \mathrm{C}$. Dans les autres cas $[20,21,34]$, la température initiale de l'eau varie de 25 à $30^{\circ} \mathrm{C}$.

Le calcul des C.O.P. thermiques et solaires présentés dans le tableau VII est fait de la façon suivante :

- pour les C.O.P. thermiques :

$$
\begin{aligned}
& \text { C.O.P.th } \text { th }_{\text {E }} /\left(Q_{\mathrm{D}}+Q_{\mathrm{CS}}\right) \\
& \text { C.O.P.th }{ }_{\text {th }}=Q_{\mathrm{G}} /\left(Q_{\mathrm{D}}+Q_{\mathrm{CS}}\right)
\end{aligned}
$$

- pour les C.O.P. solaires :

$$
\text { C.O.P.sol E }=Q_{\mathrm{E}} / Q_{\mathrm{I}} \quad \text { C.O.P. } \text { sol G }=Q_{\mathrm{G}} / Q_{\mathrm{I}} \text {. }
$$

L'efficacité du capteur est alors donnée par :

$$
\eta=\left(Q_{\mathrm{D}}+Q_{\mathrm{CS}}\right) / Q_{\mathrm{I}}=\text { C.O.P.sol E } / \text { C.O.P.th E }
$$

L'examen des différents C.O.P. solaires obtenus par les auteurs montre une variation de 0,010 (Iloeje) à 0,098 (Worsoe-Schmidt).

Le meilleur résultat, au niveau des performances globales, est obtenu par le prototype I de WorsoeSchmidt [19] mais il nous faut rappeler ici que la température initiale de l'eau est de $0^{\circ} \mathrm{C}$ ce qui tend à augmenter les C.O.P. thermique et solaire (G). Il faut noter aussi le moins bon résultat obtenu avec le prototype II : le C.O.P. solaire a été divisé par un facteur 2,7 , la production de glace étant bien inférieure à la précédente (d'un facteur 2,3).

\section{Résultats obtenus sur notre prototype de réfrigéra- teur solaire.}

Comme nous l'avons dit, le couple qui nous a paru le mieux adapté à la réfrigération solaire dans les conditions de température imposées est $\mathrm{LiCl} / \mathrm{CH}_{3} \mathrm{NH}_{2}$ avec l'équilibre suivant :

$$
\left\langle\mathrm{Li}\left(\mathrm{CH}_{3} \mathrm{NH}_{2}\right)_{2} \mathrm{Cl}\right\rangle+\left(\mathrm{CH}_{3} \mathrm{NH}_{2}\right) \rightleftarrows\left\langle\mathrm{Li}\left(\mathrm{CH}_{3} \mathrm{NH}_{2}\right)_{3} \mathrm{Cl}\right\rangle
$$

Celui-ci permet en effet l'utilisation de capteur plan à surface non sélective, la température haute nécessaire à la désorption étant de $80^{\circ} \mathrm{C}$ pour une température de condensation de $35^{\circ} \mathrm{C}$. D'autre part, l'utilisation de la méthylamine en tant que gaz réfrigérant entraîne des montages peu coûteux car les pressions de fonctionnement sont faibles et inférieures à 7 bars.

Une étude préalable sur un réacteur solide-gaz quasi-isotherme a permis d'optimiser la mise en œuvre du mélange réactionnel de façon à obtenir, dans des conditions de température données, des temps de réaction de synthèse et de décomposition compatibles avec le cycle solaire. Le tableau VIII donne les temps nécessaires pour un mélange à $40 \%$ de liant graphité et pour un tassement de 2020 moles de $\mathrm{sel} / \mathrm{m}^{3}$.

Tableau VIII. - Durées des réactions de synthèse (abs.) et de décomposition (dés.), exprimées en heures, pour différents taux de conversion $X$, pour le couple $\mathrm{LiCl} / \mathrm{CH}_{3} \mathrm{NH}_{2}$ avec nos conditions expérimentales.

\begin{tabular}{|c|c|c|c|c|c|}
\hline $\begin{array}{c}\text { Taux de } \\
\text { conversion } X\end{array}$ & 0,3 & 0,5 & 0,7 & 0,9 & 0,98 \\
\hline$t_{\mathrm{dbs} .}(\mathrm{h})$ & 0,3 & 1,2 & 3,7 & 10,8 & 15,5 \\
\hline$t_{\text {dés. }}(\mathrm{h})$ & 0,1 & 0,3 & 0,6 & 1,3 & 4 \\
\hline
\end{tabular}

[Times of the reactions of synthesis (abs.) and decomposition (des.), expressed in hours, for different conversion rates $X$, for the pair $\mathrm{LiCl} / \mathrm{CH}_{3} \mathrm{NH}_{2}$ with our experimental conditions.]

3.1 CONCEPTION DU PROTOTYPE. - Après une étude de faisabilité sur maquette [38], nous avons construit un prototype dont le schéma est présenté sur la figure 3. Il comprend :

1) le réacteur solide-gaz, situé dans le capteur solaire. Le réacteur est formé de 19 tubes en acier reliés à un collecteur tubulaire. Au centre de chaque tube, on dispose un tube coaxial perforé sur toute sa longueur pour la distribution du gaz et sur lequel on dispose des entretoises en grille d'acier inox. Neuf ailettes de support délimitent un volume donné tous les $190 \mathrm{~mm}$. Elles ont un double rôle : compartimenter de façon régulière le solide réactionnel et maintenir au centre le tube distributeur de gaz. La distance maximale sel-paroi de l'échangeur est de $1,75 \mathrm{~cm}$. Il faut comme on l'a vu précédemment respecter une distance maximale entre le sel et la paroi de l'échangeur inférieure à $2 \mathrm{~cm}$ [36], ceci afin de minimiser les problèmes de transfert de chaleur, le sel étant un matériau peu conducteur $\left(\lambda=0,1 \mathrm{~W} \cdot \mathrm{m}^{-2} \mathrm{~K}^{-1}\right)$;

2) le capteur solaire plan de surface $1,81 \mathrm{~m}^{2}$. Le coffre est en aluminium, comportant une isolation arrière de $100 \mathrm{~mm}$ de laine de verre. Ce capteur est muni de volets amovibles pour permettre le refroidissement du réacteur solide-gaz par effet cheminée. Le vitrage est simple et fixe ;

3) le condenseur à air est un tube à ailettes rondes serties en spirale. La surface d'échange totale est de $7,65 \mathrm{~m}^{2}$;

4) le collecteur est un réservoir en acier situé à la base du condenseur, d'un volume de 91 ; 

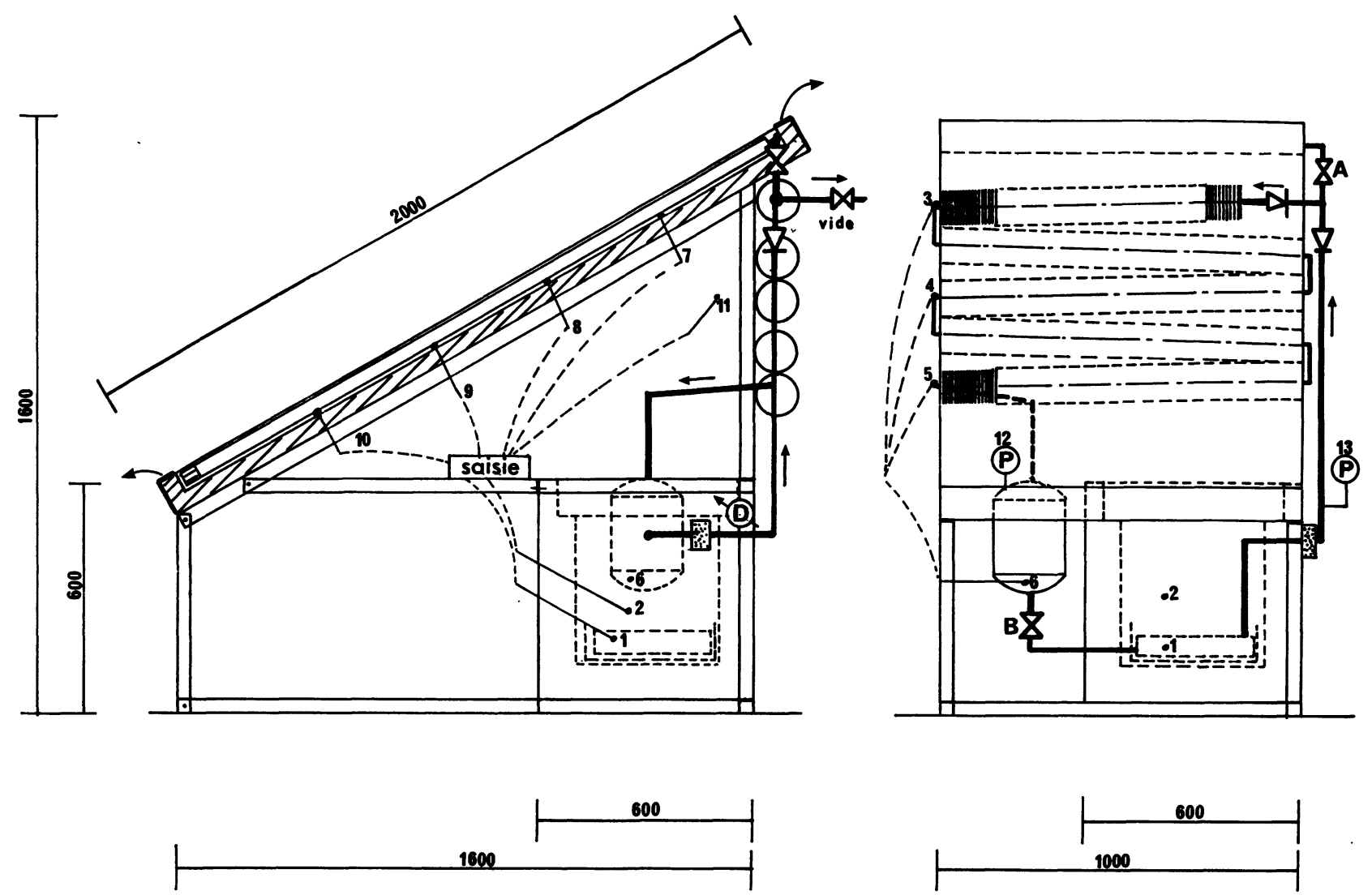

Fig. 3. - Schéma de montage du prototype de réfrigérateur solaire.

[Diagram of the prototype of the solar refrigerator.]

5) l'évaporateur est un tore en acier inox de surface d'échange en contact avec l'eau de $0,21 \mathrm{~m}^{2}$. Le volume utile pour le gaz est de 4,91;

6) le réfrigérateur a un volume utile de 39,51. L'isolation est constituée de $150 \mathrm{~mm}$ de mousse de polyuréthane et le coefficient de déperditions thermiques expérimental est de $0,335 \mathrm{~W} \cdot \mathrm{m}^{-2} \cdot \mathrm{K}^{-1}$. Un bac en aluminium placé dans le fond du réfrigérateur contient l'évaporateur et l'eau.

Deux clapets anti-retour sont disposés à la sortie du tube collecteur du réacteur solide-gaz et permettent le fonctionnement automatique du système. Dès que la pression dans le réacteur solide-gaz est supérieure de $20 \mathrm{mbar}$ à celle régnant dans le condenseur, le clapet s'ouvre et la désorption a lieu. Le fonctionnement de l'autre clapet, lors de la phase d'absorption, est identique.

Sur ce prototype, nous avons 11 mesures de température et 2 mesures de pressions, les signaux correspondant à ces mesures étant transformés et saisis séquentiellement sur un micro-ordinateur avec 5 pas de temps différents sur $24 \mathrm{~h}$. En plus, un débitmètre massique situé à la sortie de l'évaporateur permet le suivi de la cinétique d'absorption; il est relié à un enregistreur graphique.
3.2 SIMULATION DU CYCLE SOLAIRE ET RÉSULTATS EXPÉRIMENTAUX. - Les différentes expériences ont été réalisées sous insolateur artificiel, avec un éclairement moyen reçu par le capteur de $1 \mathrm{~kW} / \mathrm{m}^{2}$ qui correspond à une énergie incidente sur la durée de la désorption de 4 à $4,5 \mathrm{kWh} / \mathrm{m}^{2}$.

Le protocole expérimental est tel qu'à l'instant initial, on procède simultanément à la mise en route de l'insolateur artificiel et à la saisie des données. A la fin de la désorption qui correspond à l'arrêt du rayonnement, on ouvre les volets du capteur pour permettre le refroidissement du sel. La mise en communication du collecteur et de l'évaporateur s'effectue dès que les conditions thermodynamiques nécessaires à l'absorption sont atteintes sans tenir compte d'un écart à l'équilibre important.

La figure 4 représente l'évolution des températures et des pressions pour un cycle type de simulation. Nous allons distinguer 7 parties dans ce cycle.

Partie A. - Au cours de cette phase, la température du solide s'élève sous l'action du rayonnement sans qu'il y ait réaction chimique ; il en est de même pour la pression. A la fin de cette partie, la désorption débute.

L'hétérogénéité de l'éclairement est telle que le 

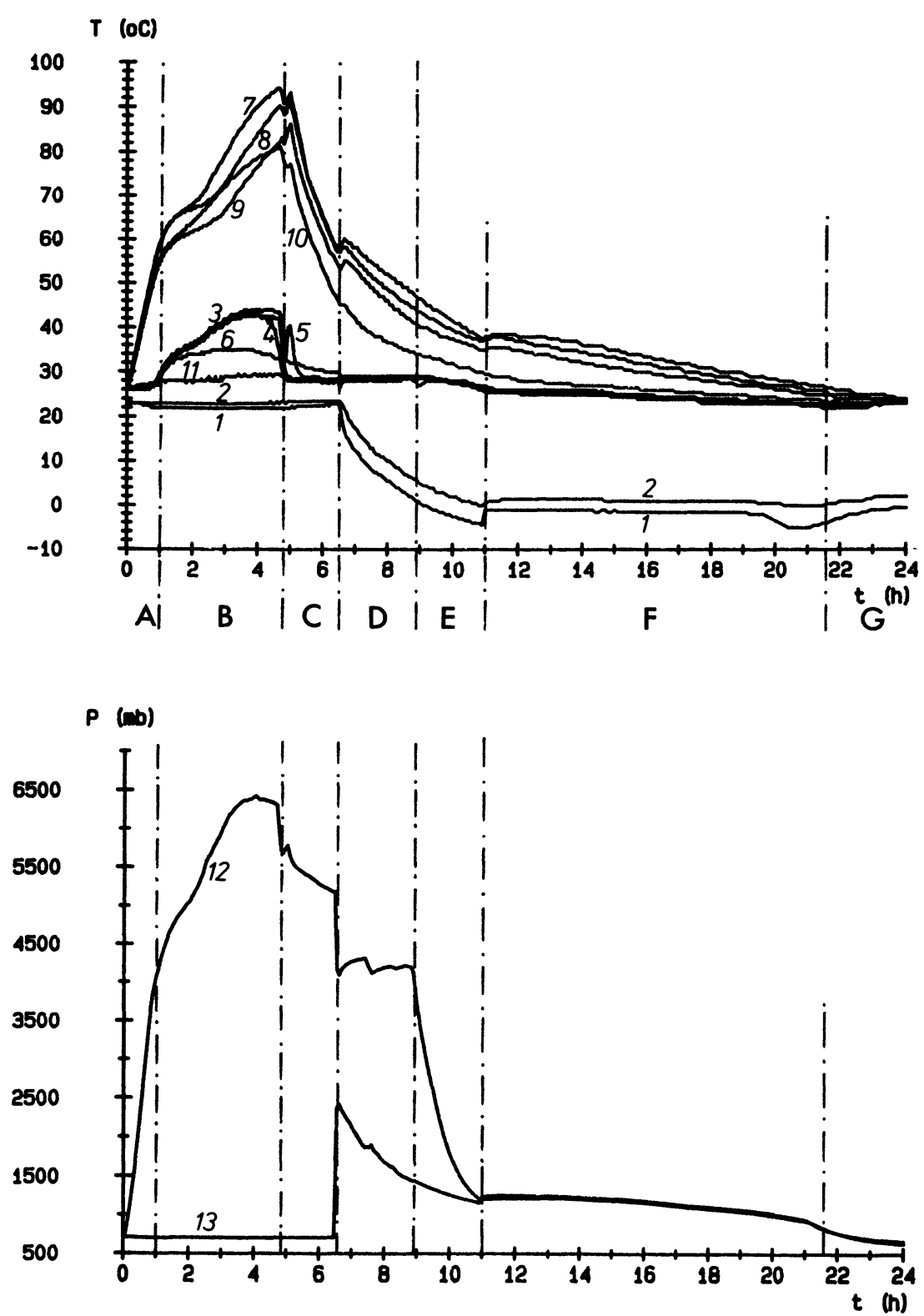

Fig. 4. - Evolution des températures et des pressions en fonction du temps pour un cycle type de simulation : 1) température de l'eau dans le réfrigérateur ; 2) température de l'air dans le réfrigérateur ; 3) température du condenseur (haut) ; 4) température du condenseur (milieu) ; 5) température du condenseur (bas) ; 6) température du collecteur ; 7) température du tube central (haut) ; 8) température du tube central (milieu haut) ; 9) température du tube central (milieu bas) ; 10) température du tube central (bas) ; 11) température ambiante ; 12) pression de condensation ; 13) pression d'évaporation.

[Temperature and pressure versus time for a typical cycle of simulation : 1) temperature of water in the refrigerator ; 2) temperature of air in the refrigerator;3) condenser temperature (high);4) condenser temperature (medium);5) condenser temperature (low); 6) collector temperature ; 7) temperature of the central tube (high) ; 8) temperature of the central tube (medium-high) ; 9) temperature of the central tube (medium-low) ; 10) temperature of the central tube (low) ; 11) ambient temperature ; 12) condensation pressure ; 13) evaporation pressure.]

tube central sur lequel sont placées les sondes de température est moins soumis au rayonnement que d'autres du fait de la position des lampes sur l'insolateur et de la distance maximale possible entre le capteur et l'insolateur $(=1 \mathrm{~m})$. Les mesures effectuées avec un pyranomètre confirment ceci. Il apparaît qu'à la fin de la zone $\mathrm{A}$, la pression indique que la désorption a lieu. Effectivement, dès le début de la partie B, les températures du tube central diminuent à cause de l'endothermicité de la désorption et simultanément les températures mesurées sur le condenseur augmentent ce qui prouve bien qu'il y 
a désorption. Or la température mesurée sur le tube est inférieure à la température minimale nécessaire pour que la désorption ait lieu, il en résulte donc que la température mesurée est différente de celle du sel.

Partie B. - C'est la phase de désorption-condensation au cours de laquelle on note une forte élévation des températures du condenseur. La pression de condensation augmente comme les températures pour atteindre à la $5^{\mathrm{e}}$ heure 6,3 bars correspondant à une température de condensation de $43^{\circ} \mathrm{C}$ (mesurée au niveau des sondes $T_{4}$ et $T_{5}$ ). Il faut noter que les températures mesurées au niveau du condenseur $\left(T_{3}\right.$ à $\left.T_{5}\right)$ sont assez élevées mais l'efficacité de celui-ci n'est pas optimale puisque les tests sont effectués à l'intérieur dans un local peu ventilé.

Le rayonnement est ensuite interrompu à la fin de la partie $B$, ce qui entraîne l'arrêt de la réaction de décomposition.

Partie C. - Dès l'arrêt de la décomposition, la pression de condensation chute rapidement puis de façon plus lente, pour atteindre à la fin de cette partie 5,2 bars. Les volets du capteur sont ouverts (début de la zone $\mathrm{C}$ ) dès l'arrêt du rayonnement et le sel commence à se refroidir comme l'indiquent les températures $T_{7}$ à $T_{10}$. Au cours de cette phase, il n'y a pas de réaction chimique, le collecteur et l'évaporateur sont isolés. A la fin de la zone $\mathrm{C}$, il faut noter le fort gradient thermique dans le sens longitudinal du tube. Ceci est dû au refroidissement par effet cheminée : la sonde située près du bas du capteur donne une température de $46^{\circ} \mathrm{C}$ et celle du haut $57^{\circ} \mathrm{C}$.

Partie D. - On relie le collecteur et l'évaporateur et le transvasement a lieu. Le fort gradient de pression entre les deux $(\Delta P=4,5$ b $)$ ainsi que la brutale évaporation du gaz due à l'absorption entraîne un fort bouillonnement du liquide dans l'évaporateur qui provoque des remontées de liquide dans la conduite de retour vers le réacteur. L'évaporateur ayant un volume insuffisant, on ferme la vanne dès que celui-ci est rempli : la pression dans le collecteur se stabilise à 4,1 bars correspondant à la température de la méthylamine liquide dans le collecteur de $29^{\circ} \mathrm{C}$.

Les conditions thermodynamiques sont telles que l'absorption peut avoir lieu dès le début de la zone $\mathrm{D}$ $(t=6,5 \mathrm{~h})$; il y a alors ouverture du clapet antiretour et l'évaporation débute entraînant une baisse des températures d'eau et d'air dans le réfrigérateur. Dès le début de l'absorption, on constate une augmentation des températures du tube central due à l'exothermicité de la réaction. A la fin de la partie $\mathrm{D}$, nous avons effectué un second et dernier transvasement, en maintenant ouverte la vanne située à la base du collecteur.

Partie E. - L'absorption se poursuit et à la suite de la mise en communication totale du collecteur et de l'évaporateur, les pressions se rejoignent à la fin de cette zone. Les températures d'eau et d'air continuent de descendre pour atteindre à la $11^{\mathrm{c}}$ heure respectivement $-4.5^{\circ}$ et $0{ }^{\circ} \mathrm{C}$, heure à laquelle a lieu l'arrêt de la surfusion, qui entraîne une accélération de la réaction par changement des conditions d'écart à l'équilibre thermodynamique [38].

Partie F. - Dans cette zone, l'évaporation a lieu à pression quasi constante $(=1200 \mathrm{mb})$ correspondant à une température de méthylamine liquide de $-2.5^{\circ} \mathrm{C}$, et les températures d'eau et d'air dans le réfrigérateur sont respectivement de $-1.5^{\circ} \mathrm{C}$ et $1^{\circ} \mathrm{C}$. A la fin de la partie $\mathrm{F}$, l'absorption est terminée. Dès la $19^{\mathrm{e}}$ heure, on observe que la totalité de l'eau présente dans le bac est transformée en glace, la température de celle-ci diminuant jusqu'à $-5^{\circ} \mathrm{C}$.

Partie G. - L'évaporateur ne contenant plus de liquide, la pression dans celui-ci baisse pour atteindre à la fin du cycle une pression de gaz résiduelle de $660 \mathrm{mb}$. Dès le début de la partie $\mathrm{G}$, la glace commence à fondre à cause des pertes thermiques, l'évaporation s'étant arrêtée.

Sur l'évolution de la composition globale $N$ du milieu au cours du temps (Fig. 5), on retrouve les parties $(D+E)$ correspondant à l'évaporation avant l'arrêt de la surfusion, $F$ correspondant à la suite de l'évaporation et $\mathrm{G}$ à l'arrêt de l'évaporation-absorption.

Les performances obtenues sont regroupées dans le tableau IX. Les C.O.P. sont calculés comme précédemment avec les valeurs suivantes obtenues sur un cycle type :

\begin{tabular}{|c|c|c|c|}
\hline$Q_{\mathrm{I}}$ & énergie incidente & $30400 \mathrm{~kJ}$ & $(100 \%)$ \\
\hline$Q_{\operatorname{cs} 1}$ & $\begin{array}{l}\text { chaleur sensible réacteur, } \\
\text { sel... }\end{array}$ & 4839 & $(16 \%)$ \\
\hline$Q_{\mathrm{D}}$ & chaleur nécessaire désorption & 5217 & $(17 \%)$ \\
\hline$Q_{\mathrm{P} 1}$ & pertes réacteur & 20344 & $(67 \%)$ \\
\hline$Q_{\mathrm{E}}$ & chaleur latente évaporation & 3243 & $(100 \%)$ \\
\hline$Q_{\mathrm{cs} 2}$ & $\begin{array}{l}\text { chaleur sensible évap., } \\
\mathrm{CH}_{3} \mathrm{NH}_{2} \ldots\end{array}$ & 1216 & $(37 \%)$ \\
\hline 26 & chaleur latente fusion glace & 1622 & $(50 \%)$ \\
\hline$Q_{\mathrm{P} 2}$ & pertes réfrigérateur & 405 & $(13 \%)$ \\
\hline
\end{tabular}

Les résultats obtenus sur ce prototype ont montré la faisabilité physico-chimique du système et l'utilisation possible du couple $\mathrm{LiCl} / \mathrm{CH}_{3} \mathrm{NH}_{2}$ dans l'objectif de la production de glace par voie solaire.

L'optimisation du taux de liant et du compactage du solide réactionnel a conduit à la détermination expérimentale de la mise en œuvre pour une utilisation solaire du système.

Par comparaison avec les résultats obtenus sur la maquette de laboratoire [38], la part de chaleur sensible au niveau du réacteur solide-gaz a fortement 


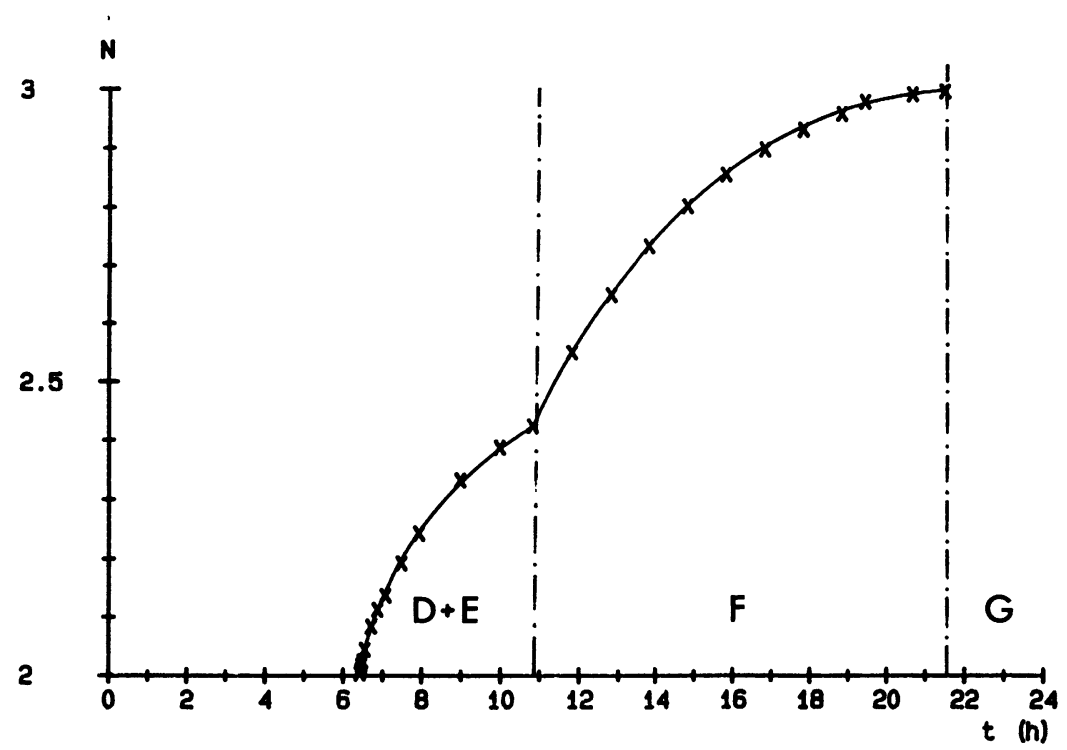

Fig. 5. - Evolution de la composition globale $N$ du milieu en fonction du temps pour le même cycle type de simulation : $N=2$ pour $\left\langle\mathrm{Li}\left(\mathrm{CH}_{3} \mathrm{NH}_{2}\right)_{2} \mathrm{Cl}\right\rangle$ et $N=3$ pour $\left\langle\mathrm{Li}\left(\mathrm{CH}_{3} \mathrm{NH}_{2}\right)_{3} \mathrm{Cl}\right\rangle$.

[Curve for the global composition $N$ of the medium versus time for a typical cycle of simulation: $N=2$ for $\left\langle\mathrm{Li}\left(\mathrm{CH}_{3} \mathrm{NH}_{2}\right)_{2} \mathrm{Cl}\right\rangle$ and $N=3$ for $\left\langle\mathrm{Li}\left(\mathrm{CH}_{3} \mathrm{NH}_{2}\right)_{3} \mathrm{Cl}\right\rangle$.]

Tableau IX. - Caractéristiques et performances de notre prototype de réfrigérateur solaire. [Characteristics and performances of our prototype of solar refrigerator.]

\begin{tabular}{|c|c|}
\hline $\begin{array}{l}\text { Couple, équilibre } \\
\text { Liant graphité (\% masse) } \\
\text { Cyclage } \\
\left.\text { Tassement (mol. sel } / \mathrm{m}^{3}\right) \\
\text { Densité énergétique }\left(\mathrm{MJ} / \mathrm{m}^{3}\right) \\
\text { Tassement max. }\left(\mathrm{mol} . \mathrm{sel} / \mathrm{m}^{3}\right) \\
\text { Densité énergétique } \max .\left(\mathrm{MJ} / \mathrm{m}^{3}\right)\end{array}$ & $\begin{array}{l}\mathrm{LiCl} / \mathrm{CH}_{3} \mathrm{NH}_{2} \text { (éq. } 2 / 3 \text { ) } \\
40 \% \\
\text { bon } \\
1930 \\
75 \\
4550 \\
187\end{array}$ \\
\hline $\begin{array}{l}\text { Echangeur du réacteur solide-gaz } \\
\text { Arrivée du gaz } \\
\text { Distance maximale sel-échangeur }(\mathrm{cm}) \\
\text { Modes de chauffage et de refroidissement } \\
\text { du réacteur solide-gaz } \\
\text { Condenseur }\left(\mathrm{S} \text { échange, } \mathrm{m}^{2}\right) \\
\left.\text { Evaporateur ( } \mathrm{S} \text { échange, } \mathrm{m}^{2}\right)\end{array}$ & $\begin{array}{l}19 \text { tubes cylindriques jointifs en acier } \\
1 \text { arrivée centrale par tube perforé }+ \text { grille } \\
1,75 \\
\text { Ch. : rayonnement artificiel } \\
\text { Re : air, convection naturelle } \\
\text { tube à ailettes, à air, conv. naturelle }(7,65) \\
\text { tore acier inox }(0,21)\end{array}$ \\
\hline $\begin{array}{l}\text { Puissance extraite évap./cycle }(\mathrm{W} / \mathrm{kg} \text { sel }) \\
\text { Durée absorption }(\mathrm{h}) \\
\text { Durée désorption }(\mathrm{h}) \\
\text { Masse de glace }(\mathrm{kg} / \mathrm{jour}) \\
\text { Masse de glace/masse de sel } \\
\text { Surface du capteur }\left(\mathrm{m}^{2}\right)\end{array}$ & $\begin{array}{l}13,5 \\
15 \\
5 \\
4,5 \\
1 \\
1,81\end{array}$ \\
\hline $\begin{array}{l}\text { C.O.P.th E } \\
\text { C.O.P.th } \\
\text { C.O.P. } \text { sul L } \\
\text { C.O.P.sol G } \\
\eta \text { capteur }\end{array}$ & $\begin{array}{l}0,322 \\
0,161 \\
0,107 \\
0,053 \\
0,33\end{array}$ \\
\hline
\end{tabular}


diminué pour passer de $45 \%$ à $16 \%$, ce qui a entraîné une augmentation du C.O.P.thE d'un facteur 1,4. Les pertes au niveau du réfrigérateur ont beaucoup diminué pour passer de $40 \%$ à $13 \%$, ceci étant dû à la meilleure isolation. Tout ceci conduit donc à une quantité de froid disponible pour la fabrication de glace plus importante : $50 \%$ au lieu de $33 \%$ pour la maquette.

Les performances obtenues sont supérieures à celles de la plupart des auteurs présentés dans cette étude mais une meilleure conception du capteur (tubes plus minces) et de l'évaporateur (plus grands volume et surface d'échange) doit permettre d'améliorer encore ces résultats.

\section{Conclusion.}

Cette étude bibliographique a montré l'intérêt suscité par la réaction chimique entre un solide et un gaz dans l'objectif de la production de froid ou de glace par voie solaire.

Après avoir maîtrisé la mise en œuvre du solide réactionnel et optimisé la structure du réacteur solide-gaz, ces deux points étant fondamentaux pour obtenir de bons résultats, quelques auteurs ont conçu des prototypes de machine à glace dont les performances moyennes sont une production quotidienne de glace de 1,5 à $4 \mathrm{~kg}$ par $\mathrm{m}^{2}$ de capteur, avec des C.O.P. solaires de 0,01 à 0,06 .

Pour notre part, après avoir choisi le couple $\mathrm{LiCl} / \mathrm{CH}_{3} \mathrm{NH}_{2}$ bien adapté à la réfrigération solaire, nous avons optimisé le mélange réactionnel par l'addition d'un liant graphité et nous avons conçu un prototype de pré-série dont les performances sont actuellement une production de glace de $4,5 \mathrm{~kg}$ par jour avec un C.O.P. solaire de 0,053. Ces résultats peuvent encore être améliorés par une meilleure conception «plus allégée » du réacteur solide-gaz.

\section{Bibliographie}

[1] Maillet D., Thèse de Docteur-Ingénieur, I.N.P. Lorraine (Nancy) Juillet 1982.

[2] Trombe F., Foex M., Production de glace à l'aide de l'énergie solaire. Colloq. Int. du C.N.R.S., Mont-Louis, L'énergie solaire : applications thermiques dans le domaine de la recherche et de l'industrie (1958, 23-28 Juin).

[3] Chinnappa J. C. V., Performance of an intermittent refrigerator operated by a flat-plate collector, Sol. Energy 6 (1962) 143-150.

[4] Duffie J. A., Chung R., Lof G. O. G., Réfrigération par absorption solaire, Colloq. Int. du C.N.R.S., Mont-Louis (1958, 23-28 Juin).

[5] Venkatesh A., Gupta M. C., Performance of an intermittent ammonia-water solar refrigerator operating with a flat plate collector, Forum Solaire Int., Vol. 1, Comples (1979).

[6] VAN PAASSEN J. P., Solar absorption refrigeration, Rapports Int., Août 1983 et Sept. 1985, Delft Univ. of Technol., Lab. for Refrig. Eng., Netherlands.

[7] Guilleminot J. J., Meunier F., Etude expérimentale d'une glacière solaire utilisant le cycle zéolithe 13X-eau, Rev. Gen. Therm. Fr. 239 (1981) 825-834.

[8] Grenier Ph., Guilleminot J. J., Mester M., Meunier F., Pons M., Entrepôt frigorifique solaire à cycle zéolithe-eau, J. Int. Transf. Hélioth. (Monastir) 1983.

[9] Pons M., Grenier Ph., Solar ice maker working with activated carbon-methanol adsorbentadsorbate pair, Congrès ISES (Montréal) Juin 1985.
[10] Boubakri A., Grenier Ph., Pons M., Utilisation du couple charbon actif-méthanol $\left(\mathrm{AC} 35-\mathrm{CH}_{3} \mathrm{OH}\right)$ pour la production de glace par énergie solaire, J. Int. Transfert Hélioth. (Rabat) 1985.

[11] PilatowsKy I., Etude sur l'utilisation du système $\mathrm{CaCl}_{2} / \mathrm{CH}_{3} \mathrm{NH}_{2}$ dans la réfrigération solaire par absorption, Thèse de $3^{\mathrm{e}}$ cycle, Univ. de Perpignan (Juillet 1978).

[12] RocA A., Réalisation d'un procédé de réfrigération utilisant le couple $\mathrm{CaCl}_{2} / \mathrm{CH}_{3} \mathrm{NH}_{2}$, Rapport de DEA, Univ. de Perpignan (Juin 1983).

[13] Buffington R. M., Absorption refrigeration with solid absorbents, Refrig. Eng. 26 (1933) 137-152.

[14] Clausen N. E., Worsoe-SchmidT P., Indoor testing of a solar-powered solid-absorption refrigerator/ice-pack freezer, Report F 77-01, May 1983, the Technical Univ. of Denmark, Refrig. Lab.

[15] Worsoe-SCHMIDT P., Solar refrigeration for developing countries using a solid-absorption cycle, Int. J. Ambient Energy 4 (1983) 115-124.

[16] Andreasen O., A finite-element solution for the absorption of ammonia in solids (To be published in Int. J. H. Mass. Transfer).

[17] WorsoE-SCHMIDT P., Some results from the development of a solid-absorption refrigerating system, CEE Absorption Heat Pumps Congress, Paris, Mars 1985, 288-300.

[18] Eggers-Lura A., Bechtoft-Nielsen P., Stubkier B., WoRSOE-SCHMIDT P., Solar powered refrigeration by intermittent solid absorption systems, Proc. Compl., Int. Meeting Heliotech. and Develop., Dharan (2-6 Nov. 1975) 83-104. 
[19] WORSOE-SCHMIDT P., A solar-powered solid-absorption refrigeration system, Int. J. Refrig. 2 (1979) 75-84.

[20] Bonauguri E., Preliminary notes on a solar absorption unit, Progr. Refrig. Sci. Technol. 2 (1969) 1411-1422.

[21] Iloeje O. C., Design construction and test run of a solar powered solid absorption refrigerator, Sol. Energy 35 (1985) 447-455.

[22] Parent Y. O., Klvana D., Mackay J., A solar energy refrigeration unit, 2nd World Cong. of Chem. Eng., Montréal 2 (1981) 210-213.

[23] KlvanA D., MADA M., VIEN A., A generator absorber model for solar-powered refrigeration units with a $\mathrm{CaCl}_{2}-\mathrm{NH}_{3}$ system, $34^{e}$ Cong. Canadien de Génie Chimique, Montréal, Oct. 1984.

[24] Bechtoft-Nielsen P., Worsoe-Schmidt P., Development of a solar-powered solid-absorption refrigeration system - Part I - Experimental investigation of the generation and absorption processes, Report F 30-77, August 1977, the Technical University of Denmark, Refrig. Lab.

[25] Heydari M. R., Pompes thermochimiques adaptées au chauffage de l'habitat. Cyclage. Modélisation et procédé d'amélioration de la cinétique de réaction, Thèse de $3^{\mathrm{e}}$ cycle, Univ. de Perpignan (Juin 1983).

[26] ANDREwS H. I., A solid-absorption machine for refrigerated railway vehicules, 8 th Cong. Int. of Refrig. (1951) 663-666.

[27] ANDREws H. I., The development of a solid-absorption refrigerating machine for use on trains, Modern Refrig. Air Control News 58 (1955) 381386.

[28] Muradov D., Shadyev O., Testing of a solar absorption refrigerator, Gelioteckhnika 7 (1971) 33-35.

[29] ShADYEV O., UMAROV G. Ya., On the temperature distribution in a solar refrigerator generator and the thermal conductivity of the adsorbant, Gelioteckhnika 8 (1972) 25-28.
[30] Flechon J., MAChizaud F., Recherche d'un couple frigorigène adapté à la réfrigération solaire en zone tropicale, Revue Phys. Appl. 14 (1979) 97105.

[31] Flechon J., Machizaud F., Réfrigération solaire par simulation en absorption sèche, Revue Phys. Appl. 15 (1980) 909-918.

[32] Flechon J., Machizaud F., Les fuites thermiques en réfrigération solaire, J. Power Sources 7 (1982) 375-390.

[33] Flechon J., Kotowski A., Machizaud F., GodMEL G., Emploi de capteurs cylindro-paraboliques pour la réfrigération dans les zones tropicales, Revue Phys. Appl. 17 (1982) 577-584.

[34] Flechon J., Machizaud F., Benhammou K., GoDMEL G., Réfrigération solaire par photothermie : premiers résultats d'un appareil réel, J. Power Sources 13 (1984) 197-216.

[35] Mauran S., Bodiot D., Crozat G., Optimisation des densités énergétiques de systèmes de stockage chimique basés sur des réactions solide/gaz renversables, Revue Phys. Appl. 18 (1983) 107-112.

[36] MAZET N., Réponse d'un réacteur solide/gaz soumis à des contraintes de pression et température : couplage entre vitesse de réaction et transferts. Application à la pompe à chaleur chimique, Thèse de $3^{\mathrm{e}}$ cycle, Univ. de Perpignan (Oct. 1984).

[37] Furrer M., EIR Bericht, n 392 (1980) 8-44.

[38] Balat M., Crozat G., Réfrigération solaire par réaction solide-gaz : faisabilité physico-chimique sur une maquette de laboratoire, Int. J. Refrig. 11 (1988) 33-40.

[39] Balat M., Crozat G., Conception et étude d'un prototype de pré-série de réfrigérateur solaire basé sur une réaction solide-gaz, Int. J. Refrig. 11 (1988) 308-314. 